Matsushima, Y. and Murakami, S.

Osaka J. Math.

2 (1965), 1-35

\title{
ON CERTAIN COHOMOLOGY GROUPS ATTACHED TO HERMITIAN SYMMETRIC SPACES
}

\author{
Yozô MATSUSHIMA and SHINGo MURAKAMI
}

(Received February 18, 1965)

This is a continuation of our previous paper [9]. In the paper [9] we have attached to a bounded symmetric domain $X$ and a discontinuous group $\Gamma$ operating on $X$ two kinds of cohomology groups. The first one is associated with a certain representation of $\Gamma$ and the second one is associated with a so-called canonical automorphic factor. The first purpose of the present paper is to prove Theorem 7.1 which establishes an isomorphism between these two kinds of cohomology groups of type $(0, q)$. This isomorphism has been proved in [9] in the case $q=\operatorname{dim}_{C} X$ as a generalization of a theorem of Eichler-Shimura, and thus Theorem 7.1 completes our previous result. Before arriving at this theorem, we shall first summarize and reformulate results in [9] in terms of Lie algebra cohomology and we shall give in $\$ 5$ a decomposition of the laplacian operators $\Delta^{\prime}, \Delta^{\prime \prime}$ into sums of the "representation parts" $\Delta_{\rho}^{\prime}$, $\Delta_{\rho}^{\prime \prime}$ and the "differential parts" $\Delta_{D}^{\prime}, \Delta_{D}^{\prime \prime}$. These decompositions of the laplacians will be utilized throughout the present paper.

The second purpose of this paper is to prove vanishing theorems for the cohomology groups in question. Our results are stated in the forms of Theorems 8.2,9.1,9.2, 12.1 and 12.2. The main idea for establishing these vanishing theorems is to relate our cohomology groups, via the decompositions of the laplacians, with the cohomology groups of the abelian Lie algebras $\mathfrak{n}^{ \pm}$. The representation parts of the laplacians are closely related to the laplacians which appeared in the paper of Kostant [8]. The cohomology theory of the Lie algebras $\mathfrak{n}^{ \pm}$is the object of Kostant's paper and we shall use his main results in the last three sections. As we shall see, one of the essential points in our proof of the vanishing theorems is in fact the vanishing of the cohomology groups of the Lie algebras $\mathfrak{n}^{ \pm}$. We note also that Theorems 9.1 and 9.2 generalize and improve the results of Calabi-Vesentini [3] and Ise [7]. It should be emphasized here that Theorems 9.1 and 9.2 are deduced, owing to the isomorphsm established by Theorem 7.1 between the two kinds of cohomologies of type $(0, q)$, from the corresponding vanishing 
theorem (Theorem 8.2) for the cohomology groups associated with a representation of $\Gamma$. Thus the study of the cohomology groups associated with a representation of $\Gamma$ is very significant in our investigaion. We mention finally that important applications of this cohomology to algebraic geometry and number theory are given recently by M. Kuga [11].

\section{Contents}

1. The complexes $A(\Gamma, X, \rho)$ and $A\left(\Gamma, X, J_{\tau}\right)$.

2. Cohomology groups of Lie algebras.

3. The complex $A(\Gamma, X, \rho)$ and the complex $C\left(\mathfrak{g}^{c}, \mathfrak{l}^{C} ; \mathcal{F}^{\prime}\right)$.

4. The bigraded complex $C\left(\mathfrak{g}^{C}, \mathfrak{l}^{C} ; \mathcal{F}\right)$.

5. Laplacian operators in $C\left(\mathfrak{g}^{C}, \mathfrak{t}^{C} ; \mathfrak{F}\right)$.

6. Harmonic cocycles in $C^{0, q}\left(\mathrm{~g}^{C}, \mathfrak{f}^{C} ; \mathcal{F}^{2}\right)$.

7. Isomorphism between the cohomology groups $H^{0, q}(\Gamma, X, \rho)$ and $H_{d \prime \prime}^{n, q}\left(\Gamma, X, J_{\mathrm{p}_{1}}\right)$.

8. Vanishing theorems for the cohomology groups $H^{0, q}(\Gamma, X, \rho)$.

9. Vanishing theorems for the cohomology groups $H_{a^{\prime \prime}}^{n, q}\left(\Gamma, X, J_{\tau}\right)$.

10. Cohomology of the abelian Lie algebras $\mathrm{n}^{ \pm}$and decomposition of the $\mathfrak{t}^{C}$ - modules $F \otimes \wedge \mathfrak{n}^{ \pm}$.

11. The operators $L^{\prime}$ and $L^{\prime \prime}$ in the module $F \otimes \wedge \mathfrak{n}^{-} \otimes \wedge \mathfrak{n}^{+}$.

12. Theorems on the cohomology groups $H^{p, q}(\Gamma, X, \rho)$.

\section{The complexes $A(\Gamma, X, \rho)$ and $A\left(\Gamma, X, J_{\tau}\right)$}

Let $G$ be a connected semisimple Lie group which has a faithful repesentation and $K$ a maximal compact subgroup of $G$. We put $X=G / K$. Let $\Gamma$ be a discrete subgroup of $G$; then $\Gamma$ is a discontinuous group operating on $X$. We assume that the quotient space $\Gamma \backslash X$ is compact or, what amounts to the same, that the quotient space $\Gamma \backslash G$ is compact. Let now $\rho$ be a representation of $G$ in a complex vector space $F$. We denote by $A^{p}(\Gamma, X, \rho)$ the vector space of all $F$-valued $C^{\infty}$-differential $p$-forms $\eta$ on $X$ such that

$$
\eta \circ L_{\gamma}=\rho(\gamma) \eta
$$

for all $\gamma \in \Gamma$, where $L_{\gamma}$ is the translation of $X$ by $\gamma$. The graded module $A(\Gamma, X, \rho)=\sum_{p} A^{p}(\Gamma, X, \rho)$ is then a complex with coboundary operator defined by the exterior differentiation $d$. We shall denote by $H^{p}(\Gamma, X, \rho)$ the cohomology groups of the complex $A(\Gamma, X, \rho)$.

We shall denote by $g$ the Lie algebra of left invariant vector fields on $G$, and by the subalgebra of $g$ corresponding to the subgroup $K$. Since every left invariant vector field on $G$ is projectable onto $\Gamma \backslash G$ by the projection $\varpi: G \rightarrow \Gamma \backslash G$, and since $\Gamma$ is a discrete subgroup of $G$, the 
mapping $\tau$ defines an injection of the Lie algebra $g$ into the Lie algebra of all vector fields on $\Gamma \backslash G$. In the following we shall identify the Lie algebra $\mathrm{g}$ with its image by this injection, so that $X \in \mathfrak{g}$ will be identified with the vector field $\varpi(X)$ on $\Gamma / G$. On the other hand, let $\langle\cdot, \cdot\rangle$ denote the Killing form of the Lie algebra $g$, and put

$$
\mathfrak{m}=\{X \in \mathfrak{g} ;\langle X, Y\rangle=0 \text { for all } Y \in \mathfrak{l}\} .
$$

We have then

$$
\mathfrak{g}=\mathfrak{m}+\mathfrak{t}, \mathfrak{m} \cap \mathfrak{t}=(0),[\mathfrak{m}, \mathfrak{m}] \subset \mathfrak{t},[\mathfrak{t}, \mathfrak{m}] \subset \mathfrak{m} .
$$

We shall denote by $\mathfrak{g}^{c}$ the complexification of the Lie algebra $g$ and by $\mathfrak{l}^{C}$ and $\mathfrak{m}^{C}$ the complex vector subspaces of $\mathrm{g}^{C}$ spanned by and $\mathfrak{m}$ respectively. Obviously $\mathfrak{t}^{C}$ is a subalgebra of $\mathfrak{g}^{C}$.

Let $\eta$ be a form in $A^{p}(\Gamma, X, \rho)$. Denoting by $\pi$ the projection of $G$ onto $X$, we define a form $\eta^{0}$ on $G$ by putting $\eta_{s}^{0}=\rho\left(s^{-1}\right)(\eta \circ \pi)_{s}(s \in G)$. The form $\eta^{0}$ is invariant under left translations of $G$ by elements of $\Gamma$, and therefore $\eta^{0}$ is regarded as a form on the quotient space $\Gamma \backslash G$. The image $A_{0}^{p}(\Gamma, X, \rho)$ of $A^{p}(\Gamma, X, \rho)$ by the mapping $\eta \rightarrow \eta^{0}$ consists of all $F$-valued $p$-forms on $\Gamma \backslash G$ which satisfiy the conditions

$$
\left\{\begin{array}{l}
\theta(X) \eta^{0}+\rho(X) \eta^{0}=0 \\
i(X) \eta^{0}=0
\end{array}\right.
$$

for all $X \in \mathfrak{f}$, where $\theta(X)$ and $i(X)$ denote the operators of Lie derivation and interior product by $X$ respectively. Moreover, the exterior differentiation $d$ in $A(\Gamma, X, \rho)$ is transformed to the operator $d$ for $\eta^{0}$ such that

$$
\left(d \eta^{0}\right)\left(X_{1}, \cdots, X_{p+1}\right)=\sum_{u=1}^{n+1}(-1)^{u_{+1}}\left(X_{u}+\rho\left(X_{u}\right)\right) \eta^{0}\left(X_{1}, \cdots, \grave{X}_{u}, \cdots, X_{p+1}\right)
$$

for $X_{1}, \cdots, X_{\eta+1} \in \mathfrak{m}[9$, Part I $\$ 4]$. We remark that each form $\eta^{0} \in$ $A_{0}^{p}(\Gamma, X, \rho)$ defines canonically a $p$-linear alternating form on $\mathrm{g}^{C}$ with values in the vector space $\mathcal{F}$ of all $C^{\infty}$-functions on $\Gamma \backslash G$; this form determines $\eta^{0}$ and is characterized among $\mathcal{F}_{\text {-valued }} p$-linear alternating forms on $\mathrm{g}^{c}$ as the one satisfying (1.2) for all $X \in \mathfrak{l}^{c}$ when $\theta(X)$ and $i(X)$ are interpreted in a suitable way (see $\$ 2$ ).

In this paper we shall be concerned with the case where $X=G / K$ is a symmetric bounded domain in $C^{N}$. Since $G$ is assumed to have a faithful representation, there exists a complex form $G^{C}$ of $G$, that is, a complex Lie group with Lie algebra $\mathrm{g}^{c}$ which has $G$ as a real analytic subgroup corresponding to $\mathrm{g} \subset \mathrm{g}^{C}$. The complex subgroup of $G^{C}$ corresponding to the subalgebra $\mathfrak{f}^{C}$ will be denoted by $K^{c}$. In this case, the module $A(\Gamma, X, \rho)$ is bigraded; the subspace $A^{p, q}(\Gamma, X, \rho)$ consists of the 
forms $\eta \in A^{p+q}(\Gamma, X, \rho)$ which are of type $(p, q)$. We know that $H^{r}(\Gamma, X, \rho)$ decomposes into the direct sum $\sum_{p+q=r} H^{p, q}(\Gamma, X, \rho)$ where $H^{p, q}(\Gamma, X, \rho)$ is the subgroup of $H^{r}(\Gamma, X, \rho)$ consisting of the elements represented by closed forms of type $(p, q)[9$, p. 405].

Let $A_{0}^{p, q}(\Gamma, X, \rho)$ be the submodule of $A_{0}^{p+q}(\Gamma, X, \rho)$ corresponding to the submodule $A^{p, q}(\Gamma, X, \rho)$ of $A^{p+q}(\Gamma, X, \rho)$ by the isomorphism $\eta \rightarrow \eta^{0}$. To characterize the forms belonging to $A_{0}^{p, q}(\Gamma, X, \rho)$, we recall the following facts [9, p. 395]. The vector space $\mathfrak{m}^{c}$ decomposes canonically into the direct sum $\mathfrak{m}^{C}=\mathfrak{n}^{+}+\mathfrak{n}^{-}$where $\mathfrak{n}^{+}$and $\mathfrak{n}^{-}$are abelian subalgebras of $\mathfrak{g}^{C}$ such that $\left[\mathfrak{f}^{C}, \mathfrak{n}^{+}\right] \subset \mathfrak{n}^{+}$and $\left[\mathfrak{l}^{C}, \mathfrak{n}^{-}\right] \subset \mathfrak{n}^{-}$; moreover, $\mathfrak{f}$ contains a Cartan subalgebra $\mathfrak{h}$ of $g$ and, in the set of roots of $\mathfrak{g}^{c}$ with respect to the Cartan subalgebra $\mathfrak{h}^{c}$, there is a subset $\Psi$ (of the so-called positive complementary roots) such that $\mathfrak{n}^{+}$(resp. $\mathfrak{n}^{-}$) is spanned by $\left\{X_{\alpha} ; \alpha \in \Psi\right\}$ (resp. $\left\{X_{\bar{\alpha}} ; \alpha \in \Psi\right\}$ ), where $X_{a}$ (resp. $X_{\bar{\alpha}}$ ) are eigenvectors of the roots $\alpha$ (resp. $-\alpha$ ). We can choose these vectors so that $\left\langle X_{\alpha}, X_{\bar{\alpha}}\right\rangle=1$ for all $\alpha \in \Psi$. We have then $X_{\bar{\alpha}}=\bar{X}_{\alpha}$, where - denotes the conjugation of $\mathrm{g}^{C}$ with respect to $g$. Using these notations, a form $\eta^{0} \in A_{0}^{p+q}(\Gamma, X, \rho)$ belongs to $A_{0}^{p, q}(\Gamma, X, \rho)$ if and only if the following condition is verified; if $\eta^{0}\left(X_{1}, \cdots, X_{p+q}\right) \neq 0$ with $X_{i} \in \mathfrak{n}^{ \pm}$, then the number of $X_{i}$ belonging to $\mathfrak{n}^{+}$(resp. to $\mathfrak{n}^{-}$) equals $p$ (resp. q). [9, p. 400].

We have defined in $[9$, p. 397] the canonical automorphic factor $J$ on $X=G / K$. $J$ is a $C^{\infty}$-mapping of $G \times X$ into $K^{C}$ such that

1) $J(s, x)$ is holomorphic in $x \in X$;

2) $J(s t, x)=J(s, t x) J(t, x)$ for $s, t \in G, x \in X$.

For a holomorphic representation $\tau$ of $K^{c}$ in a complex vector space $S$ we put

$$
J_{\tau}(s, x)=\tau(J(s, x)) .
$$

Then $J_{\tau}$ is a GL $(S)$-valued automorphic factor which we call the (canonical) automorphic factor of type $\tau$. Let now $A^{p, q}\left(\Gamma, X, J_{\tau}\right)$ (resp. $\left.A^{r}\left(\Gamma, X, J_{\tau}\right)\right)$ be the vector space of all $S$-valued forms $\eta$ of type $(p, q)$ (resp. of degree $r$ ) on $X$ such that

$$
\left(\eta \circ L_{\gamma}\right)_{x}=J_{\tau}(\gamma, x) \eta_{x}
$$

for all $x \in X$ and $\gamma \in \Gamma$. We have $A^{r}\left(\Gamma, X, J_{\tau}\right)=\sum_{\eta_{+q}=r} A^{p, q}\left(\Gamma, X, J_{\tau}\right)$. Put $A\left(\Gamma, X, J_{\tau}\right)=\sum_{p, q} A^{p, q}\left(\Gamma, X, J_{\tau}\right)$. The operator $d^{\prime \prime}$ defines a coboundary operator of type $(0,1)$ in the bigraded complex $A\left(\Gamma, X, J_{\tau}\right)$. We shall denote by $H_{a^{\prime \prime}}^{p, q}\left(\Gamma, X, J_{\tau}\right)$ the cohomology groups of this complex $A\left(\Gamma, X, J_{\tau}\right)$ with the coboundary operator $d^{\prime \prime}$.

As in the case of $A^{p}(\Gamma, X, \rho)$, the module $A^{p, q}\left(\Gamma, X, J_{\tau}\right)$ corresponds to a module $A_{0}^{p, q}\left(\Gamma, X, J_{\tau}\right)$ of $(p+q)$-forms on $\Gamma \backslash G$; for a form $\eta \in A^{p, q}\left(\Gamma, X, J_{\tau}\right)$ 
we define a $(p+q)$-form $\eta^{0}$ on $G$ by putting $\eta_{s}^{0}=J_{\tau}\left(s, x_{0}\right)^{-1}(\eta \circ \pi)_{s}$, where $x_{0}=\pi(e), e$ being the identity element of $G$. Then $\eta^{0}$ is induced by the projecton $\varpi: G \rightarrow \Gamma \backslash G$ from an $S$-valued form on $\Gamma \backslash G$, which we denote also by $\eta^{0}$. The mapping $\eta \rightarrow \eta^{0}$ maps the module $A^{p, q}\left(\Gamma, X, J_{\tau}\right)$ bijectively onto the module $A_{0}^{p, q}\left(\Gamma, X, J_{\tau}\right)$ consisting of all $S$-valued $(p+q)$-forms on $\Gamma \backslash G$ such that

$$
\left\{\begin{array}{l}
\theta(X) \eta^{0}+\tau(X) \eta^{0}=0 \\
i(X) \eta^{0}=0
\end{array}\right.
$$

for all $X \in \mathfrak{l}^{C}$, and that if $\eta^{0}\left(X_{1}, \cdots, X_{p+q}\right) \neq 0$ with $X_{i} \in \mathfrak{n}^{ \pm}$, then the number of $X_{i}$ belonging to $\mathfrak{n}^{+}$(resp. to $\mathfrak{n}^{-}$) equals $p$ (resp. $q$ ). Moreover, the operator $d^{\prime \prime}$ on $A\left(\Gamma, X, J_{\tau}\right)$ is transformed to the operator $d^{\prime \prime}$ on $A_{0}\left(\Gamma, X, J_{\tau}\right)$ $=\sum_{p, q} A_{0}^{\eta, q}\left(\Gamma, X, J_{\tau}\right)$ such that if $\eta^{0} \in A_{0}^{p, q}\left(\Gamma, X, J_{\tau}\right)$

$$
\begin{aligned}
& \left(d^{\prime \prime} \eta^{0}\right)\left(X_{\alpha_{1}}, \cdots, X_{\alpha_{p}}, X_{\bar{\beta}_{1}}, \cdots, X_{\bar{\beta}_{q+1}}\right) \\
& \quad=\sum_{u=1}^{q+1}(-1)^{p+u-1} X_{\bar{\beta}_{u}} \eta^{0}\left(X_{\alpha_{1}}, \cdots, X_{\alpha_{p}}, X_{\bar{\beta}_{1}}, \cdots,{X_{\bar{\beta}_{u}}}, \cdots, X_{\bar{\beta}_{q+1}}\right)
\end{aligned}
$$

where $X_{\alpha_{i}}$ (resp. $X_{\bar{\beta}_{j}}$ ) are elements of the basis of $\mathfrak{n}^{+}$(resp. $\mathfrak{n}^{-}$).

We have studied in [9] the cohomology groups $H^{p}(\Gamma, X, \rho)$, $H_{d^{\prime \prime}}^{p, q}\left(\Gamma, X, J_{\tau}\right)$ as well as some relations among these cohomology groups, by applying the theory of vector bundle-valued harmonic forms. Some formulae established in [9] will be needed in this paper, and will be recalled in the following sections.

\section{Cohomology groups of Lie algebras}

In this section we shall summarize some notions in the cohomology theory of Lie algebras which we shall use in the following sections. Let $\mathrm{g}^{C}$ be a complex Lie algebra and $\mathfrak{h}^{C}$ a subalgebra of $\mathrm{g}^{C}$. Assume that a representation $m$ of $\mathrm{g}^{C}$ in a complex vector space $V$ be given; the vector space $V$ is then regarded as a $\mathrm{g}^{c}$-module. We shall denote by $C^{p}\left(\mathfrak{g}^{c} ; V\right)$ the vector space of all $p$-linear alternating forms on $\mathrm{g}^{C}$ with values in $V$. Put $C\left(\mathfrak{g}^{c} ; V\right)=\sum_{p} C^{p}\left(\mathfrak{g}^{c} ; V\right)$. In this graded module $C\left(\mathrm{~g}^{C} ; V\right)$ we can define the operators $i(X)$ and $\mathscr{L}(X)$ of interior product and Lie derivation by $X ; \mathscr{L}(X)$ is the degree-preserving operator defined by

$$
\begin{aligned}
(\mathscr{L}(X) \eta)\left(X_{1}, \cdots, X_{p}\right)= & \left.m(X) \eta\left(X_{1}, \cdots, X_{p}\right)\right) \\
& -\sum_{u=1}^{p} \eta\left(X_{1}, \cdots,\left[X, X_{u}\right], \cdots, X_{p}\right)
\end{aligned}
$$

for $\eta \in C^{p}\left(\mathfrak{g}^{c} ; V\right)(p>0)$ and $\varphi(X) \eta=m(X) \eta$ for $\eta \in C^{0}\left(\mathfrak{g}^{C} ; V\right)(=V)$, while $i(X)$ is the operator of degree -1 defined by

$$
(i(X) \eta)\left(X_{1}, \cdots, X_{p-1}\right)=\eta\left(X, X_{1}, \cdots, X_{p-1}\right)
$$


for $\eta \in C^{p}\left(g^{C} ; V\right)(p>0)$ and $i(X) \eta=0$ for $\eta \in C^{0}\left(g^{0} ; V\right)$. There exists one and only one operator $d$ of degree 1 such that $i(X) d+d i(X)=\mathscr{L}(X)$ and $d$ is given by

$$
\begin{gathered}
(d \eta)\left(X_{1}, \cdots, X_{p+1}\right)=\sum_{u=1}^{p+1}(-1)^{u+1} m\left(X_{u}\right) \eta\left(X_{1}, \cdots, \hat{X}_{u}, \cdots, X_{p+1}\right) \\
+\sum_{u<v}(-1)^{u+v} \eta\left(\left[X_{u}, X_{v}\right], X_{1}, \cdots, \ddot{X}_{u}, \cdots, \hat{X}_{v}, \cdots, X_{p+1}\right)
\end{gathered}
$$

Since $d^{2}=0$, the module $C\left(\mathfrak{g}^{c} ; V\right)$ is a complex with $d$ as coboundary operator. We shall call this complex the cochain complex of $\mathfrak{g}^{C}$ with coefficients in the $\mathrm{g}^{C}$-module $V$. The cohomology groups of this complex will be denoted by $H^{p}\left(\mathfrak{g}^{C} ; V\right)$.

Let $C^{p}\left(\mathfrak{g}^{C}, \mathfrak{l}^{C} ; V\right)$ be the submodule of $C^{p}\left(\mathrm{~g}^{C} ; V\right)$ consisting of all elements $\eta \in C^{p}\left(\mathfrak{g}^{C} ; V\right)$ such that $\mathscr{L}(X) \eta=i(X) \eta=0$ for all $X \in \mathfrak{l}^{C}$. The submodule $C\left(\mathfrak{g}^{C}, \mathfrak{f}^{C} ; V\right)=\sum_{p} C^{p}\left(\mathfrak{g}^{C}, \mathfrak{l}^{C} ; V\right)$ is stable under the operator $d$ and thus a subcomplex of $C\left(\mathfrak{g}^{C} ; V\right)$. We shall call this complex $C\left(\mathfrak{g}^{C}, \mathfrak{l}^{C} ; V\right)$ the cochain complex of $\mathrm{g}^{C}$ relative to with coefficients in $V$, and the cohomology groups of this complex will be denoted by $H^{p}\left(\mathfrak{g}^{C}, \mathfrak{t}^{C} ; V\right)$.

We note that if $\mathrm{g}^{C}$ is decomposed into the direct sum $\mathrm{g}^{C}=\mathfrak{t}^{C}+\mathfrak{m}^{C}$ as vector space and if $\left[\mathfrak{l}^{C}, \mathrm{~m}^{c}\right] \subset \mathrm{m}^{c}$, then each cochain $\eta \in C^{p}\left(\mathfrak{g}^{c}, \mathfrak{l}^{c} ; V\right)$ is identified with a $p$-linear alternating form $\eta$ on $\mathrm{m}^{c}$ such that $\mathscr{L}(X) \eta=0$ for all $X \in \mathfrak{l}^{C}$, where $\mathscr{L}(X)$ is defined by the same formula as (2.1). Therefore, if $\mathrm{m}^{*}$ denotes the dual vector space of $\mathrm{m}^{c}$, the module $C^{p}\left(\mathfrak{g}^{C}, \mathfrak{l}^{C} ; V\right)$ may be canonically identified with the subspace of $V \otimes \bigwedge^{p} \mathfrak{m}^{*}$ consisting of all elements of $V \otimes \wedge^{p} \mathrm{~m}^{*}$ which are annihilated by the operators $m(Y) \otimes 1+1 \otimes \operatorname{ad}^{*}(Y)$ for all $Y \in k^{C}$, where $\operatorname{ad}^{*}$ is the representation of $\mathfrak{t}^{C}$ in $\bigwedge^{p} \mathrm{~m}^{*}$ induced by the adjoint representation of $\mathrm{g}^{C}$.

\section{The complex $A(\Gamma, X, \rho)$ and the complex $C\left(g^{C}, \mathfrak{l}^{C} ; F\right)$}

We retain the notation in $\$ 1$ and resume here some of the results in [9] in the language of the cohomology theory of Lie algebra. Let $\mathcal{R}$ be the vector space of all complex valued $C^{\infty}$-functions on $\Gamma \backslash G$ and let $\mathcal{F}=\mathscr{R} \otimes_{\boldsymbol{c}} F$. We may identify $\mathcal{F}$ with the vector space of all $F$-valued $C^{\infty}$-functions on $\Gamma \backslash G$. The vector spaces $\mathscr{R}$ and $F$ have structures of $\mathrm{g}^{C}$-module in an obvious way and hence so does the tensor product $\mathcal{F}_{\mathrm{r}}$ by the rule

$$
m(X)(f \otimes u)=X f \otimes u+f \otimes \rho(X) u,
$$

where $X \in \mathrm{g}^{c}, f \in \mathcal{R}$ and $u \in F$. The vector space $\mathcal{F}$ has also two other $\mathrm{g}^{C}$-module structures defined respectively by

$$
m_{1}(X)(f \otimes u)=X f \otimes u
$$


and

$$
m_{2}(X)(f \otimes u)=f \otimes \rho(X) u .
$$

We denote by $\mathscr{F}_{i}$ the vector space $\mathcal{F}$ with the $\mathrm{g}^{C}$-module structure $m_{i}(i=1,2)$, while we denote simply by $\mathcal{F}$ the vector space $\mathcal{F}$ with the $\mathrm{g}^{C}$-module structure $m$.

Let $C\left(\mathfrak{g}^{C} ; \mathcal{F}_{1}\right)=\sum_{p} C^{p}\left(\mathfrak{g}^{C} ; \mathcal{F}_{1}\right)$ (resp. $\left.C\left(\mathfrak{g}^{C} ; \mathscr{F}_{i}\right)\right)$ be the cochain complex of the Lie algebra $\mathrm{g}^{C}$ with coefficients in the $\mathrm{g}^{C}$-module $\mathcal{F}_{\text {(resp. }} \mathcal{F}_{i}$ ). We denote by $d$ (resp. $d_{i}$ ) the coboundary operator in $C\left(\mathrm{~g}^{C} ; \mathcal{F}_{1}\right.$ ) (resp. $C\left(\mathrm{~g}^{C} ; \mathcal{F}_{i}\right)$ ) and by $i(X)$ and $\mathscr{L}(X)$ (resp. $\mathscr{L}_{i}(X)$ ) with $X \in \mathrm{g}^{C}$ the operators of interior product and Lie derivation in $C\left(\mathrm{~g}^{C} ; \mathcal{F}_{1}\right)\left(\operatorname{resp} . C\left(\mathrm{~g}^{C} ; \mathcal{F}_{i}\right)\right)$. In the following we shall consider the operators $d_{i}$ and $\mathscr{L}_{i}(X)$ as operators in $C\left(g^{C} ; \mathcal{F}\right)$.

Let $C\left(\mathfrak{g}^{C}, \mathfrak{l}^{C} ; \mathcal{F}\right)$ be the cochain complex of $\mathfrak{g}^{C}$ relative to $\mathfrak{l}^{C}$ with

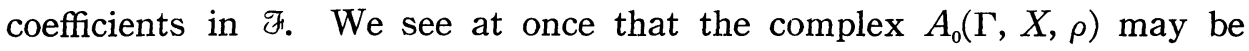
identified with the complex $C\left(\mathfrak{g}^{C}, \mathfrak{l}^{C} ; \mathcal{F}_{1}\right)$. Therefore, by the results in $\S 1$, there is a canonical isomorphism of the complex $A(\Gamma, X, \rho)$ onto the complex $C\left(\mathfrak{g}^{C}, \mathfrak{t}^{C} ; F_{1}\right)$. We shall identity by this isomorphism the complex $A(\Gamma, X, \rho)$ with $C\left(\mathfrak{g}^{C}, \mathfrak{l}^{C} ; \mathscr{F}\right)$ and therefore the cohomology groups $H^{p}(\Gamma, X, \rho)$ with the relative cohomology groups $H^{p}\left(\mathfrak{g}^{C}, \mathfrak{l}^{C} ; \mathcal{F}\right)$. Note that the complex $C\left(\mathrm{~g}^{C} ; \mathscr{H}_{1}\right)$ is identified with the complex of all $F$-valued $C^{\infty}$-differential forms on $\Gamma \backslash G$ for which the coboundary operator is the operator of exterior differentiation.

A positive definite hermitian inner product $(\eta, \zeta)$ in $C^{p}\left(\mathfrak{g}^{C}, \mathfrak{l}^{C} ; \mathcal{F}\right)$ is defined by the formula

$$
(\eta, \zeta)=\frac{1}{p !} \sum_{i_{1}, \cdots, i_{p}=1}^{N} \int_{\Gamma \backslash G}\left(\eta\left(X_{i_{1}}, \cdots, X_{i_{p}}\right), \zeta\left(X_{i_{1}}, \cdots, X_{i_{p}}\right)\right)_{F} d v
$$

where $\left\{X_{1}, \cdots, X_{N}\right\}$ is a basis of $m$ which is orthonormal with respect to the Killing form of $\mathrm{g}$ and $(,)_{F}$ denotes an inner product in $F$ satisfying the condition of $[9, \mathrm{p} .375]$. We extend this inner product to $C\left(\mathfrak{g}^{C}, \mathfrak{l}^{C} ; \mathcal{F}_{1}\right)$ by the condition that $C^{p}\left(\mathfrak{g}^{C}, \mathfrak{f}^{C} ; \mathcal{F}_{1}\right)$ and $C^{q}\left(\mathfrak{g}^{C}, \mathfrak{l}^{C} ; \mathcal{F}_{1}\right)$ are orthogonal for $p \neq q$. There is an operator $\delta$ of degree -1 in $C\left(\mathfrak{g}^{C}, \mathfrak{t}^{C} ; \mathcal{F}^{\prime}\right)$ which is adjoint to $d$ with respect to this inner product. If $\eta \in C^{p}\left(\mathfrak{g}^{C}, \mathfrak{l}^{c} ; \mathcal{F}_{1}\right)$ we have, for $Y_{1}, \cdots, Y_{p+1} \in \mathfrak{m}^{C}$,

$$
\left\{\begin{array}{l}
(d \eta)\left(Y_{1}, \cdots, Y_{p+1}\right)=\sum_{u=1}^{n+1}(-1)^{u+1}\left(m_{1}\left(Y_{u}\right)+m_{2}\left(Y_{u}\right)\right)\left(\eta\left(Y_{1}, \cdots, \hat{Y}_{u}, \cdots, Y_{p+1}\right)\right) \\
(\delta \eta)\left(Y_{1}, \cdots, Y_{p-1}\right)=-\sum_{k=1}^{N}\left(m_{1}\left(X_{k}\right)-m_{2}\left(X_{k}\right)\right)\left(\left(i\left(X_{k}\right) \eta\right)\left(Y_{1}, \cdots, Y_{p-1}\right)\right)
\end{array}\right.
$$

We define the "laplacian" operator $\Delta$ by $\Delta=d \delta+\delta d$ and we call a cocycle $\eta \in C^{p}\left(\mathfrak{g}^{c}, \mathfrak{f}^{C} ; \mathcal{F}\right)$ harmonic if $\Delta \eta=0$. Then every cohomology 
class in the cohomology group $H^{p}\left(\mathfrak{g}^{C}, \mathfrak{l}^{C} ; \mathscr{F}^{-}\right)$is represented by a unique harmonic cocycle [9, p. 383].

The "covariant differential" $D \eta$ of a cochain $\eta \in C^{p}\left(\mathfrak{g}^{c}, \mathfrak{f}^{c} ; \mathscr{F}^{\prime}\right)$ is the cochain in $C^{p+1}\left(\mathfrak{g}^{c}, \mathfrak{l}^{c} ; \mathscr{F}\right)$ defined by

$$
(D \eta)\left(Y_{1}, \cdots, Y_{p+1}\right)=\left(d_{1} \eta\right)\left(Y_{1}^{\mathfrak{m}}, \cdots, Y_{p+1}^{\mathfrak{m}}\right)
$$

for $Y_{1}, \cdots, Y_{p^{+1}} \in \mathrm{g}^{c}$, where $Y_{i}^{\mathfrak{m}}$ denotes the $\mathrm{m}^{c}$-component of $Y_{i}$ with respect to the decomposition (1.1). There is an operator $D_{*}$ of $C\left(\mathrm{~g}^{c}, \mathfrak{l}^{C} ; \mathcal{G}\right)$ of degree -1 which is adjoint to $D[9, \mathrm{p}$. 387]. If $\eta \in$ $C^{p}\left(\mathfrak{g}^{c}, \mathfrak{f l}^{C} ; \mathfrak{F}\right)$, we have

$$
\left\{\begin{array}{l}
(D \eta)\left(Y_{1}, \cdots, Y_{p+1}\right)=\sum_{u=1}^{p+1}(-1)^{u_{+1}} m_{1}\left(Y_{u}\right)\left(\eta\left(Y_{1}, \cdots, \hat{Y}_{u}, \cdots, Y_{p+1}\right)\right) \\
\left(D_{*} \eta\right)\left(Y_{1}, \cdots, Y_{p-1}\right)=-\sum_{k=1}^{N} m_{1}\left(X_{k}\right)\left(\left(i\left(X_{k}\right) \eta\right)\left(Y_{1}, \cdots, Y_{p-1}\right)\right)
\end{array}\right.
$$

where $Y_{1}, \cdots, Y_{p+1} \in \mathfrak{m}^{c}$.

We put

$$
d_{\rho}=d-D, \quad \delta_{\rho}=\delta-D_{*} .
$$

Then $d_{\rho}$ and $\delta_{\rho}$ are operators in $C\left(\mathfrak{g}^{C}, \mathfrak{f}^{C} ; F_{1}\right)$ of degree 1 and of degree -1 respectively and they are adjoint to each other. We get from (3.1) and (3.2)

$$
\left\{\begin{array}{l}
\left(d_{p} \eta\right)\left(Y_{1}, \cdots, Y_{p+1}\right)=\sum_{u=1}^{p+1}(-1)^{u_{+1}} m_{2}\left(Y_{u}\right)\left(\eta\left(Y_{1}, \cdots, \hat{Y}_{u}, \cdots, Y_{p+1}\right)\right) \\
\left(\delta_{p} \eta\right)\left(Y_{1}, \cdots, Y_{p-1}\right)=\sum_{k=1}^{N} m_{2}\left(X_{k}\right)\left(\left(i\left(X_{k}\right) \eta\right)\left(Y_{1}, \cdots, Y_{p-1}\right)\right)
\end{array}\right.
$$

where $Y_{1}, \cdots, Y_{p+1} \in \mathfrak{m}^{c}$.

\section{The bigraded complex $C\left(\mathfrak{g}^{C}, \mathfrak{f}^{C} ; \mathcal{F}^{\prime}\right)$}

From now on we assume that $X=G / K$ is a symmetric bounded domain in $C^{N}$. Since $\mathfrak{m}^{C}$ is the direct sum of $\mathfrak{n}^{+}$and $\mathfrak{n}^{-}$(cf. $\S 1$ ), the complex $C\left(\mathfrak{g}^{C}, \mathfrak{l}^{C} ; \mathcal{F}\right)$ is then bigraded; the submodule $C^{p, q}\left(\mathfrak{g}^{c}, \mathfrak{l}^{C} ; \mathscr{F}\right)$ of $C\left(\mathfrak{g}^{C}, \mathfrak{f}^{C} ; \mathscr{F}^{\prime}\right)$ consists of all $\eta \in C^{r}\left(\mathfrak{g}^{C}, \mathfrak{k}^{C} ; \mathscr{F}^{\prime}\right)(r=p+q)$ such that if $\eta\left(Y_{1}\right.$, $\left.\cdots, Y_{r}\right) \neq 0$ and $Y_{i} \in \mathfrak{n}^{ \pm}$for $i=1, \cdots, r$, then the number of $Y_{i}$ belonging to $\mathfrak{n}^{+}$(resp. to $\mathfrak{n}^{-}$) equals $p$ (resp. $q$ ). We see that $C^{p, q}\left(\mathfrak{g}^{C}, \mathfrak{l}^{C} ; \mathscr{F}^{\prime}\right)$ is precisely the submodule of $C\left(\mathfrak{g}^{C}, \mathfrak{t}^{C} ; \mathscr{F}^{\prime}\right)$ which is identified with the submodule $A^{p, q}(\Gamma, X, \rho)$ of $A(\Gamma, X, \rho)$ by the idenfication of $A(\Gamma, X, \rho)$

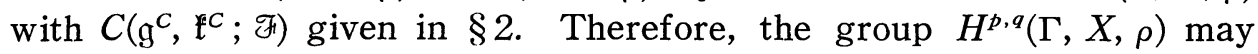
be identified with the subgroup $H^{p, q}\left(\mathfrak{g}^{C}, \mathfrak{l}^{C} ; \mathcal{F}^{\prime}\right)$ of $H^{p+q}\left(\mathfrak{g}^{C}, \mathfrak{f}^{C} ; \mathcal{F}^{\prime}\right)$ consisting of the cohomology classes represented by the cocycles in $C^{p, q}\left(\mathfrak{g}^{C}, \mathfrak{l}^{C} ; \mathscr{F}\right)$.

The operators $d, D$, and $d_{\rho}$ in the bigraded complex $C\left(\mathfrak{g}^{C}, \mathfrak{l}^{C} ; \mathbb{F}^{\prime}\right)$ decompose into the direct sums

$$
d=d^{\prime}+d^{\prime \prime}, \quad D=D^{\prime}+D^{\prime \prime}, \quad d_{\rho}=d_{\rho}^{\prime}+d_{\rho}^{\prime \prime}
$$


where $d^{\prime}, D^{\prime}$ and $d_{\rho}^{\prime}$ (resp. $d^{\prime \prime}, D^{\prime \prime}$ and $d_{\rho}^{\prime \prime}$ ) are operators of type $(1,0)$ (resp. of type $(0,1))$. There exist the adjoint operators of $d^{\prime}, D^{\prime}, d_{\rho}^{\prime}$ (resp. of $\left.d^{\prime \prime}, D^{\prime \prime}, d_{\rho}^{\prime \prime}\right)$ which are of type $(-1,0)$ (resp. of type $(0,-1)$ ) and they will be denoted by $\delta^{\prime}, D_{*}^{\prime}, \delta_{\rho}^{\prime}\left(\operatorname{resp} . \delta^{\prime \prime}, D_{*}^{\prime \prime}, \delta_{\rho}^{\prime \prime}\right)$. We have then

$$
\begin{array}{ll}
d^{\prime}=D^{\prime}+d_{\rho}^{\prime}, & d^{\prime \prime}=D^{\prime \prime}+d_{\rho}^{\prime \prime}, \\
\delta^{\prime}=D_{*}^{\prime}+\delta_{\rho}^{\prime}, & \delta^{\prime \prime}=D_{*}^{\prime \prime}+\delta_{\rho}^{\prime \prime} .
\end{array}
$$

We now identify the module $C^{p, q}\left(\mathfrak{g}^{C}, \mathfrak{l}^{C} ; \mathcal{F}\right)$ with a subspace of $\mathscr{F} \otimes \bigwedge^{p} \mathfrak{n}^{-} \otimes \bigwedge^{q} \mathfrak{n}^{+}$in the following way. Let $\Psi=\left\{\alpha_{1}, \cdots, \alpha_{N}\right\}$ be the set of the positive complementary roots and let $\left\{X_{\alpha} ; \alpha \in \Psi\right\}$ and $\left\{X_{\bar{\alpha}} ; \alpha \in \Psi\right\}$ be the eigenvectors of roots as introduced in $\S 1$. To simplify the notation, we put henceforth

$$
X_{i}=X_{\alpha_{i}}, \quad X_{\bar{i}}=X_{\bar{\alpha}_{i}}
$$

for $\alpha_{i} \in \Psi, i=1, \cdots, N$. Since $\left\{X_{i}\right\}$ and $\left\{X_{\bar{i}}\right\}$ are bases of $\mathfrak{n}^{+}$and $\mathfrak{n}^{-}$ respectively and since $\left\langle X_{i}, X_{j}\right\rangle=\delta_{i j}$, one of the vector spaces $\mathfrak{n}^{+}$and $\mathfrak{n}^{-}$ may be identified with the dual of the other. By the remark at the end of $\S 2$, we can then define an injection $i: C^{p, q}\left(\mathfrak{g}^{c}, \mathfrak{l} \mathfrak{l}^{c} ; \mathcal{F}\right) \rightarrow \mathscr{F} \otimes \bigwedge^{p} \mathfrak{n}^{-} \otimes \bigwedge^{q} \mathfrak{n}^{+}$ by putting

$$
i(\eta)=\sum_{i_{1},<\cdots<i_{p}} \sum_{\bar{j}_{1}<\cdots<\bar{j}_{q}} \eta\left(X_{i_{1}}, \cdots, X_{i_{p}}, X_{\bar{j}_{1}}, \cdots, X_{\bar{j}_{q}}\right) \otimes \bigwedge_{t=1}^{p} X_{\bar{i}_{t}} \otimes \bigwedge_{s=1}^{q} X_{j_{s}} .
$$

Let us denote by $\operatorname{ad}_{-}^{p}\left(\right.$ resp. $\left.\operatorname{ad}_{+}^{q}\right)$ or simply by ad $\left(\operatorname{resp}_{-} \mathrm{ad}_{+}\right)$the representation of $\mathfrak{l}^{C}$ in $\bigwedge^{p} \mathfrak{n}^{-}$(resp. in $\bigwedge^{q} \mathfrak{n}^{+}$) induced by the adjoint action of $\mathfrak{l}^{C}$ in $\mathrm{g}^{C}$. Then, the image of $i$ coincides with the subspace of $\mathfrak{F} \otimes \bigwedge^{p} \mathfrak{n}^{-} \otimes \bigwedge^{a} \mathfrak{n}^{+}$consisting of all element $\eta$ such that

$$
\left(m(Y) \otimes 1 \otimes 1+1 \otimes \operatorname{ad}_{-}^{p}(Y) \otimes 1+1 \otimes 1 \otimes \operatorname{ad}_{+}^{q}(Y)\right) \eta=0
$$

for all $Y \in \mathfrak{l}^{C}$. In the following we shall identify the module $C^{p, q}\left(\mathfrak{g}^{C}, \mathfrak{l}^{C} ; \mathbb{F}^{\prime}\right)$ with its image by $i$ in $\mathscr{F} \otimes \bigwedge^{p} \mathfrak{n}^{-} \otimes \bigwedge^{q} \mathfrak{n}^{+}$.

Under this identification and notation, we get from (3.1), (3.2) and (3.3) the following formulae for the operators in $C\left(\mathfrak{g}^{C}, \mathfrak{l}^{C} ;\right.$ F $)$.

$$
\left\{\begin{array}{l}
D^{\prime}=\sum_{k=1}^{N} m_{1}\left(X_{k}\right) \otimes \varepsilon\left(X_{\bar{k}}\right) \otimes 1, \quad D_{*}^{\prime}=-\sum_{k=1}^{N} m_{1}\left(X_{\bar{k}}\right) \otimes i\left(X_{k}\right) \otimes 1, \\
d_{\rho}^{\prime}=\sum_{k=1}^{N} m_{2}\left(X_{k}\right) \otimes \varepsilon\left(X_{\bar{k}}\right) \otimes 1, \quad \delta_{\rho}^{\prime}=\sum_{k=1}^{N} m_{2}\left(X_{\bar{k}}\right) \otimes i\left(X_{k}\right) \otimes 1, \\
D^{\prime \prime}=w_{1} \sum_{k=1}^{N} m_{1}\left(X_{\bar{k}}\right) \otimes 1 \otimes \varepsilon\left(X_{k}\right), \quad D_{*}^{\prime \prime}=-w_{1} \sum_{k=1}^{N} m_{1}\left(X_{k}\right) \otimes 1 \otimes i\left(X_{\bar{k}}\right), \\
d_{\rho}^{\prime \prime}=w_{1} \sum_{k=1}^{N} m_{2}\left(X_{\bar{k}}\right) \otimes 1 \otimes \varepsilon\left(X_{k}\right), \quad \delta_{\rho}^{\prime \prime}=w_{1} \sum_{k=1}^{N} m_{2}\left(X_{k}\right) \otimes 1 \otimes i\left(X_{\bar{k}}\right) .
\end{array}\right.
$$

Here $w_{1}$ is the operator on $\mathscr{F} \otimes \wedge \mathfrak{n}^{-} \otimes \wedge \mathfrak{n}^{+}$defined by $w_{1}\left(\sum_{p} f \otimes c_{p} \otimes c^{\prime}\right)$ 
$=\Sigma_{p}(-1)^{p} f \otimes c_{p} \otimes c^{\prime}$, where $f \in \mathcal{F}, c_{p} \in \bigwedge^{p} \mathfrak{n}^{-}$and $c^{\prime} \in \bigwedge \mathfrak{n}^{+} ; \varepsilon(X)$ is the operator of exterior multiplication by $X$, and $i\left(X_{k}\right)$ (resp. $i\left(X_{\bar{k}}\right)$ ) is the operator on $\wedge \mathfrak{n}^{-}$(resp. $\left.\wedge \mathfrak{n}^{+}\right)$defined as usual by the formula

$$
i\left(X_{k}\right)\left(Z_{1} \wedge \cdots \wedge Z_{p}\right)=\sum_{u=1}^{p}(-1)^{u-1}\left\langle X_{k}, Z_{u}\right\rangle Z_{1} \wedge \cdots \wedge Z_{u} \wedge \cdots \wedge Z_{p}
$$

for $Z_{1}, \cdots, Z_{p} \in \mathfrak{n}^{-}$. It follows follows from [9, p. 411] that an element of $C\left(\mathfrak{g}^{C}, \mathfrak{l}^{C} ; \mathfrak{F}^{\prime}\right)$ is harmonic if and only if it is annihilated by all these operators (4.2). In the next section we shall give another proof of this fact.

We choose a basis $\left\{Y_{1}, \cdots, Y_{r}\right\}$ of such that

$$
\left\langle Y_{a}, Y_{b}\right\rangle=-\delta_{a b} \quad(a, b=1, \cdots, r),
$$

and put

$$
\left[X_{i}, X_{\bar{j}}\right]=\sum_{a=1}^{r} c_{a i \bar{j}} Y_{a} \quad(i, j=1, \cdots, N) .
$$

From the relation $\left\langle\left[X_{i}, Y_{a}\right], X_{\bar{j}}\right\rangle+\left\langle Y_{a},\left[X_{i}, X_{j}\right]\right\rangle=0$, we have

$$
\left[Y_{a}, X_{i}\right]=-\sum_{j=1}^{N} c_{a i j} X_{j} .
$$

Here and in the following, the index $a$ will range over $1, \cdots, r$, and the indices $k, j, i$ will range over $1, \cdots, N$ unless otherwise stated.

For later use, we refer the following obvious formulae.

$$
\left\{\begin{array}{l}
\operatorname{ad}_{+}\left(Y_{a}\right)=\sum_{k=1}^{N} \varepsilon\left(\left[Y_{a}, X_{k}\right]\right) i\left(X_{\bar{k}}\right), \\
\operatorname{ad}_{-}\left(Y_{a}\right)=\sum_{k=1}^{N} \varepsilon\left(\left[Y_{a}, X_{k}\right]\right) i\left(X_{k}\right) .
\end{array}\right.
$$

Lemma 4. 1. We have

$$
\begin{array}{ll}
\sum_{a=1}^{r} \operatorname{ad}_{+}^{q}\left(Y_{a}\right)^{2}=-\sum_{k=1}^{N} \operatorname{ad}_{+}^{q}\left(\left[X_{k}, X_{k}\right]\right)=-\frac{q}{2} 1 & \text { on } \bigwedge_{\mathfrak{n}^{+}}, \\
\sum_{a=1}^{r} \operatorname{ad}_{-}^{p}\left(Y_{a}\right)^{2}=\sum_{k=1}^{N} \operatorname{ad}_{-}^{p}\left(\left[X_{k}, X_{\bar{k}}\right]\right)=-\frac{p}{2} 1 \quad \text { on } \bigwedge_{\mathfrak{n}^{-},}^{p}
\end{array}
$$

where 1 denotes the identity mapping.

Proof. We shall prove the first formula; the second one is proved quite analogously. We have

$$
\begin{aligned}
& \sum_{a=1}^{r} \operatorname{ad}_{+}^{q}\left(Y_{a}\right)^{2}\left(X_{i_{1}} \wedge \cdots \wedge X_{i_{q}}\right) \\
& \quad=\sum_{a=1}^{r} \sum_{u=1}^{q}\left(X_{i_{1}} \wedge \cdots \wedge\left[Y_{a},\left[Y_{a}, X_{i_{u}}\right]\right) \wedge \cdots \wedge X_{i_{q}}\right) \\
& \quad+\sum_{a=1}^{r} \sum_{u, v, u \neq v}\left(X_{i_{1}} \wedge \cdots \wedge\left[Y_{a}, X_{i_{u}}\right] \wedge \cdots \wedge\left[Y_{a}, X_{i_{v}}\right] \wedge \cdots \wedge X_{i_{q}}\right) .
\end{aligned}
$$

By (4.4) the second term on the right side is written in the form

$$
\sum_{u, v, u \neq v} \sum_{j, k} R_{i_{\mu} j \bar{k} i_{v}}\left(X_{i_{1}} \wedge \cdots \wedge \check{\mathscr{X}}_{j} \wedge \cdots \wedge \stackrel{\tilde{X}}{k}_{k} \wedge \cdots \wedge X_{i_{q}}\right),
$$


where we put

$$
R_{\boldsymbol{i}_{\boldsymbol{u}} \bar{j} \bar{k} \boldsymbol{i}_{\boldsymbol{v}}}=\sum_{a=1}^{r} c_{a \boldsymbol{i}_{\boldsymbol{u}} \bar{j}} c_{\boldsymbol{a \boldsymbol { i } _ { \boldsymbol { v } } \overline { k }}}
$$

Put

$$
a_{u v}=\sum_{j, k} R_{i_{u} j k i v} X_{j} \wedge X_{k}
$$

and we claim $a_{u v}=0$. Indeed, by Bianchi identity $R_{i_{u} j_{k i} i_{v}}+R_{i_{u} \bar{k} i_{v}}=0$, we get $R_{i_{u} \bar{j} \bar{k} i_{v}}=R_{i_{u} \bar{k} \bar{j}_{i_{v}}}$. Therefore $a_{u v}=\sum_{j, k} R_{i_{u} \bar{k} \bar{j}_{i}} X_{j} \wedge X_{k}=-\sum_{j_{, k}} R_{i_{u} \bar{k} \bar{j}_{i}} X_{k} \wedge X_{j}$ $=-a_{u v}$, whence $a_{u v}=0$. It follows that the second term on the right side of (4.6) vanishes. On the other hand, we have

$$
\sum_{a=1}^{r}\left[Y_{a},\left[Y_{a}, X_{i}\right]\right]=-\sum_{k, j} R_{i \bar{j} j k} X_{k}
$$

By Bianchi identity $R_{i \bar{j} j \bar{k}}+R_{i \bar{k} \bar{j} j}=0$, we get $R_{i j}{ }_{j \bar{k}}=-R_{i \bar{k}^{j} j}=\sum_{a=1}^{r} c_{a i \bar{k}} c_{a \bar{j}_{j}}$. Then, we get by (4.3) and (4.4)

$$
\begin{aligned}
& \sum_{a}\left[Y_{a},\left[Y_{a}, X_{i}\right]\right]=\sum_{j}\left(\sum_{a} c_{a j \bar{j}} \sum_{k} c_{a i \bar{k}} X_{k}\right) \\
& =\sum_{j}\left(\sum_{a} c_{a j \bar{j}}\left(-\left[Y_{a}, X_{i}\right]\right)\right)=-\sum_{j}\left[\left[X_{j}, X_{\bar{j}}\right], X_{i}\right] .
\end{aligned}
$$

It follows from this and (4.6) that

$$
\sum_{a} \operatorname{ad}_{+}^{q}\left(Y_{a}\right)^{2}\left(X_{i_{1}} \wedge \cdots \wedge X_{i q}\right)=-\sum_{k} \operatorname{ad}_{+}^{q}\left(\left[X_{k}, X_{k}\right]\right)\left(X_{i_{1}} \wedge \cdots \wedge X_{i_{q}}\right) .
$$

The both sides are equal to $-\frac{q}{2}\left(X_{i_{1}} \wedge \cdots \wedge X_{i_{q}}\right)$ as follows from (4.6) and (4.7), since we know that $\sum_{j} R_{i j} \bar{j}_{j}=-\frac{1}{2} \delta_{i k}$. This proves Lemma 4. 1.

We put

$$
s=\alpha_{1}+\cdots+\alpha_{N}
$$

For a linear form $\lambda$ on the Cartan subalgebra $\mathfrak{h}^{C}$ of $\mathfrak{g}^{C}$, we shall denote as usual also by $\lambda$ the vector $H_{\lambda} \in \mathfrak{h}^{C}$ such that $\lambda(H)=\left\langle H_{\lambda}, H\right\rangle$ for all $H \in \mathfrak{h}^{c}$; the inner product $\langle\lambda, \mu\rangle$ between two linear forms $\lambda$, $\mu$ means the value $\left\langle H_{\lambda}, H_{\mu}\right\rangle$. It is well known that the vector $s$ of $\mathfrak{h}^{c}$ belongs to the center of $[2]$.

Lemma 4. 2. The element $\left[X_{i}, X_{i}\right]$ is equal to the vector $\alpha_{i} \in \mathfrak{h}^{C}$, and therefore $\sum_{i=1}^{N}\left[X_{i}, X_{\bar{i}}\right]=s$. Moreover, we have

$$
\left\langle s, \alpha_{i}\right\rangle=\frac{1}{2} \quad(i=1, \cdots, N) .
$$

Proof. Since $\left\langle X_{i}, X_{\bar{i}}\right\rangle=1$, we have $\left\langle\left[X_{i}, X_{\bar{i}}\right], H\right\rangle=\left\langle X_{\bar{i}},\left[H, X_{i}\right]\right\rangle$ $=\alpha_{i}(H)$ for all $H \in \mathfrak{h}^{C}$, which proves the first assertion. Since $s=$ $\sum_{i=1}^{N}\left[X_{i}, X_{i}\right]$, we have

$$
\left\langle\alpha_{i}, s\right\rangle X_{i}=\alpha_{i}\left(\sum_{i=1}^{N}\left[X_{i}, X_{\bar{i}}\right]\right) X_{i}=\operatorname{ad}_{+}^{1}\left(\sum_{i=1}^{N}\left[X_{i}, X_{\bar{i}}\right]\right) X_{i}=\frac{1}{2} X_{i}
$$


by Lemma 4. 1. Thus $\left\langle s, \alpha_{i}\right\rangle=\left\langle\alpha_{i}, s\right\rangle=\frac{1}{2}$.

\section{Laplacian operators in $C\left(\mathfrak{g}^{C}, \mathfrak{f}^{C} ; \mathcal{F}\right)$}

Besides the laplacian $\Delta=d \delta+\delta d$, we define the following laplacians which are all bidegree-preserving operators in $C\left(\mathfrak{g}^{C}, \mathfrak{t}^{C} ; \mathcal{F}_{1}\right)$.

$$
\begin{array}{ll}
\Delta^{\prime}=d^{\prime} \delta^{\prime}+\delta^{\prime} d^{\prime}, & \Delta^{\prime \prime}=d^{\prime \prime} \delta^{\prime \prime}+\delta^{\prime \prime} d^{\prime \prime}, \\
\Delta_{D}^{\prime}=D^{\prime} D_{*}^{\prime}+D_{*}^{\prime} D^{\prime}, & \Delta_{D}^{\prime \prime}=D^{\prime \prime} D_{*}^{\prime \prime}+D_{*}^{\prime \prime} D^{\prime \prime}, \\
\Delta_{\rho}^{\prime}=d_{\rho}^{\prime} \delta_{\rho}^{\prime}+\delta_{\rho}^{\prime} d_{\rho}^{\prime}, & \Delta_{\rho}^{\prime \prime}=d_{\rho}^{\prime \prime} \delta_{\rho}^{\prime \prime}+\delta_{\rho}^{\prime \prime} d_{\rho}^{\prime \prime} .
\end{array}
$$

We know that $\Delta=\Delta^{\prime}+\Delta^{\prime \prime}[9$, p. 404]. From the formula (4.2) and (4.5) we get easily the following formulae for these laplącians :

$$
\left\{\begin{array}{l}
\Delta_{D}^{\prime}=-\sum_{k=1}^{N} m_{1}\left(X_{k}\right) m_{1}\left(X_{k}\right) \otimes 1 \otimes 1-\sum_{a} m_{1}\left(Y_{a}\right) \otimes \operatorname{ad}_{-}\left(Y_{a}\right) \otimes 1, \\
\Delta_{\rho}^{\prime}=\sum_{k=1}^{N} m_{2}\left(X_{\bar{k}}\right) m_{2}\left(X_{k}\right) \otimes 1 \otimes 1+\sum_{a} m_{2}\left(Y_{a}\right) \otimes \mathrm{ad}_{-}\left(Y_{a}\right) \otimes 1, \\
\Delta_{D}^{\prime \prime}=-\sum_{k=1}^{N} m_{1}\left(X_{k}\right) m_{1}\left(X_{\bar{k}}\right) \otimes 1 \otimes 1-\sum_{a} m_{1}\left(Y_{a}\right) \otimes 1 \otimes \mathrm{ad}_{+}\left(Y_{a}\right), \\
\Delta_{\rho}^{\prime \prime}=\sum_{k=1}^{N} m_{2}\left(X_{k}\right) m_{2}\left(X_{\bar{k}}\right) \otimes 1 \otimes 1+\sum_{a} m_{2}\left(Y_{a}\right) \otimes 1 \otimes \operatorname{ad}_{+}\left(Y_{a}\right) .
\end{array}\right.
$$

It follows from these formulas and those of $[9, \mathrm{p} .405]$ the following equalities :

$$
\begin{aligned}
& \Delta^{\prime}=\Delta_{D}^{\prime}+\Delta_{\rho}^{\prime}+\sum_{k=1}^{N}\left(m_{2}\left(X_{\bar{k}}\right) m_{1}\left(X_{k}\right)-m_{1}\left(X_{k}\right) m_{2}\left(X_{k}\right)\right) \otimes 1 \otimes 1 \\
& \Delta^{\prime \prime}=\Delta_{D}^{\prime \prime}+\Delta_{\rho}^{\prime \prime}+\sum_{k=1}^{N}\left(m_{2}\left(X_{k}\right) m_{1}\left(X_{\bar{k}}\right)-m_{1}\left(X_{k}\right) m_{2}\left(X_{\bar{k}}\right)\right) \otimes 1 \otimes 1
\end{aligned}
$$

Since $m_{1}(X)$ and $m_{2}\left(X^{\prime}\right)$ commute for any $X$ and $X^{\prime}$ in $\mathrm{g}^{c}$, we have

$$
\Delta=\Delta^{\prime}+\Delta^{\prime \prime}=\left(\Delta_{D}^{\prime}+\Delta_{D}^{\prime \prime}\right)+\left(\Delta_{\rho}^{\prime}+\Delta_{\rho}^{\prime \prime}\right) \text {. }
$$

Therefore, $(\Delta \eta, \eta)=\left(\Delta_{D}^{\prime} \eta, \eta\right)+\left(\Delta_{D}^{\prime \prime}, \eta\right)+\left(\Delta_{\rho}^{\prime} \eta, \eta\right)+\left(\Delta_{\rho}^{\prime \prime} \eta, \eta\right)$ and each term of the right side is non-negative. Therefore, if we have $\Delta \eta=0$, then each term vanishes and this implies in turn that $\eta$ is annihilated by the operators $d_{\rho}^{\prime}, \delta_{\rho}^{\prime}, d^{\prime \prime}, \delta_{\rho}^{\prime \prime}, D^{\prime}, D_{*}^{\prime}, D^{\prime \prime}$ and $D_{*}^{\prime \prime}$. The coverse being also true, we get the following

Lemma 5. 1. An element $\eta$ of $C\left(\mathfrak{g}^{C}, \mathfrak{l}^{C} ; \mathcal{F}_{1}\right)$ is harmonic if and only if $\Delta_{\rho}^{\prime} \eta=\Delta_{\rho}^{\prime \prime} \eta=\Delta_{D}^{\prime} \eta=\Delta_{D}^{\prime \prime} \eta=0$.

Let now $C$ be the element of the universal envelopping algebra $\mathscr{E}$ of $\mathrm{g}^{C}$ defined by

$$
C=\sum_{k=1}^{N}\left(X_{k} X_{\bar{k}}+X_{\bar{k}} X_{k}\right)-\sum_{a} Y_{a}^{2}
$$

The element $C$ is called the Casimir element of $\&$ and is in the center of $\mathscr{E}$. The $\mathrm{g}^{C}$-module structures of $\mathcal{F}_{\text {}}$ are extended naturally to $\mathscr{E}$-module structures of $\mathcal{F}$ and we have 


$$
m_{i}(C)=\sum_{k=1}^{N}\left\{m_{i}\left(X_{k}\right) m_{i}\left(X_{\bar{k}}\right)+m_{i}\left(X_{\bar{k}}\right) m_{i}\left(X_{k}\right)\right\}-\sum_{a} m_{i}\left(Y_{a}\right)^{2} \quad(i=1,2) .
$$

Therefore

$$
2 \sum_{k=1}^{N} m_{i}\left(X_{\bar{k}}\right) m_{i}\left(X_{k}\right)=m_{i}(C)+\sum_{k=1}^{N} m_{i}\left(\left[X_{\bar{k}}, X_{k}\right]\right)+\sum_{a} m_{i}\left(Y_{a}\right)^{2} .
$$

On the other hand, we have

$$
\begin{aligned}
& 2 \sum_{a} m_{i}\left(Y_{a}\right) \otimes \mathrm{ad}_{-}\left(Y_{a}\right)= \\
& \quad=\sum_{a}\left(m_{i} \otimes \mathrm{ad}_{-}\right)\left(Y_{a}\right)^{2}-\sum_{a} m_{i}\left(Y_{a}\right)^{2} \otimes 1-\sum_{a} 1 \otimes \operatorname{ad}_{-}\left(Y_{a}\right)^{2} \\
& \quad=\sum_{a}\left(m_{\imath} \otimes \mathrm{ad}_{-}\right)\left(Y_{a}\right)^{2}-\sum_{a} m_{i}\left(Y_{a}\right)^{2} \otimes 1-\sum_{a} 1 \otimes \operatorname{ad}_{-}\left(\left[X_{k}, X_{k}\right]\right)
\end{aligned}
$$

where the last equality follows by Lemma 4.1 and $m_{i} \otimes a d_{\text {- denotes the }}$ tensor product of representations $m_{i}$ and ad.. Using these, we get from (5.1) the following formulae for the laplacians :

$$
\begin{aligned}
2 \Delta_{D}^{\prime}= & -\left\{m_{1}(C) \otimes 1+\sum_{a}\left(m_{1} \otimes \mathrm{ad}_{-}\right)\left(Y_{a}\right)^{2}\right. \\
& \left.+\sum_{k=1}^{N}\left(m_{1} \otimes \mathrm{ad}_{-}\right)\left(\left[X_{\bar{k}}, X_{k}\right]\right)\right\} \otimes 1 \\
2 \Delta_{\rho}^{\prime}= & \left\{m_{2}(C) \otimes 1+\sum_{a}\left(m_{2} \otimes \mathrm{ad}_{-}\right)\left(Y_{a}\right)^{2}\right. \\
& \left.+\sum_{k=1}^{N}\left(m_{2} \otimes \mathrm{ad}_{-}\right)\left(\left[X_{\bar{k}}, X_{k}\right]\right)\right\} \otimes 1 .
\end{aligned}
$$

Analogously, we have also

$$
\begin{aligned}
2 \Delta_{D}^{\prime \prime}= & -t^{-1} \circ\left[\left\{m_{1}(C) \otimes 1+\sum_{a}\left(m_{1} \otimes \mathrm{ad}_{+}\right)\left(Y_{a}\right)^{2}\right.\right. \\
& \left.\left.+\sum_{k=1}^{N}\left(m_{1} \otimes \mathrm{ad}_{+}\right)\left(\left[X_{k}, X_{\bar{k}}\right]\right)\right\} \otimes 1\right] \circ t \\
2 \Delta_{\rho}^{\prime \prime}= & t^{-1} \circ\left[\left\{m_{2}(C) \otimes 1+\sum_{a}\left(m_{2} \otimes \mathrm{ad}_{+}\right)\left(Y_{a}\right)^{2}\right.\right. \\
& \left.\left.+\sum_{k=1}^{N}\left(m_{2} \otimes \mathrm{ad}_{+}\right)\left(\left[X_{k}, X_{\bar{k}}\right]\right)\right\} \otimes 1\right] \circ t .
\end{aligned}
$$

Here $t$ denotes the canonical isomorphism of $\mathscr{F} \otimes \wedge \mathfrak{n}^{-} \otimes \wedge \mathfrak{n}^{+}$onto $\mathfrak{H} \otimes \wedge \mathfrak{n}^{+} \otimes \wedge \mathfrak{n}^{-}$defined by $t(f \otimes u \otimes v)=f \otimes v \otimes u$.

\section{Harmonic cocycles in $C^{0, q}\left(\mathfrak{g}^{C}, \mathfrak{l}^{C} ; \mathfrak{F}\right)$}

The vector space $C^{0, q}\left(\mathfrak{g}^{c}, \mathfrak{l}^{c} ; \mathscr{F}^{\prime}\right)$ is identified with the subspace of $\mathscr{F} \otimes \bigwedge^{a} \mathfrak{n}^{+}$consisting of all $\eta \in \mathscr{H} \otimes \stackrel{a}{\wedge} \mathfrak{n}^{+}$such that

$$
\left(m_{1}(Y) \otimes 1+m_{2}(Y) \otimes 1+1 \otimes \operatorname{ad}_{+}(Y)\right) \eta=0
$$

for all $Y \in \mathfrak{l}^{c}$, since $\operatorname{dim}_{C} \AA \mathfrak{n}^{-}=1$ and $\operatorname{ad}_{-}^{0}(Y)=0$. Moreover, we see from (5.1) that

$$
\left\{\begin{array}{l}
\Delta_{D}^{\prime}=-\sum_{k=1}^{N} m_{1}\left(X_{\bar{k}}\right) m_{1}\left(X_{k}\right) \otimes 1 \\
\Delta_{\rho}^{\prime}=\sum_{k=1}^{N} m_{2}\left(X_{\bar{k}}\right) m_{2}\left(X_{k}\right) \otimes 1
\end{array}\right.
$$

and 


$$
\begin{aligned}
& \Delta_{D}^{\prime \prime}-\Delta_{D}^{\prime}=-\sum_{k=1}^{N} m_{1}\left(\left[X_{k}, X_{\bar{k}}\right]\right) \otimes 1-\sum_{a} m_{1}\left(Y_{a}\right) \otimes \operatorname{ad}_{+}\left(Y_{a}\right) \\
& \Delta_{\rho}^{\prime \prime}-\Delta_{\rho}^{\prime}=\sum_{k=2}^{N} m_{2}\left(\left[X_{k}, X_{\bar{k}}\right]\right) \otimes 1+\sum_{a} m_{2}\left(Y_{a}\right) \otimes \operatorname{ad}_{+}\left(Y_{a}\right)
\end{aligned}
$$

hold on $C^{0, q}\left(\mathfrak{g}, \mathfrak{f} ; F_{1}\right)$. Since $\left[X_{k}, X_{\bar{k}}\right]$ and $Y_{a}$ belong to $\mathfrak{l}^{C}$, we may apply (6.1) with $Y=\left[X_{k}, X_{\bar{k}}\right]$ and $Y=Y_{a}$. Then

$$
\begin{aligned}
& \left(\Delta_{D}^{\prime \prime}-\Delta_{D}^{\prime}\right)-\left(\Delta_{\rho}^{\prime \prime}-\Delta_{\rho}^{\prime}\right) \\
& \quad=\sum_{k} 1 \otimes \operatorname{ad}_{+}\left(\left[X_{k}, X_{\bar{k}}\right]\right)+\sum_{a}\left(1 \otimes \operatorname{ad}_{+}\left(Y_{a}\right)^{2}\right)=0,
\end{aligned}
$$

where the last equality follows by Lemma 4.1. We get therefore

$$
\Delta_{D}^{\prime \prime}+\Delta_{\rho}^{\prime}=\Delta_{D}^{\prime}+\Delta_{\rho}^{\prime \prime} \text {. }
$$

on $C^{0, q}\left(\mathfrak{g}, \mathfrak{f} ; \mathcal{F}^{\prime}\right)$. Let now $\eta \in C^{0, q}\left(\mathfrak{g}^{C}, \mathfrak{l}^{C} ; \mathcal{F}^{\prime}\right)$. Then we have

$$
\left(\Delta_{D}^{\prime \prime} \eta, \eta\right)+\left(\Delta_{\rho}^{\prime} \eta, \eta\right)=\left(\Delta_{D}^{\prime} \eta, \eta\right)+\left(\Delta_{\rho}^{\prime \prime} \eta, \eta\right)
$$

and hence $\Delta_{D}^{\prime \prime} \eta=\Delta_{\rho}^{\prime} \eta=0$ implies $\Delta_{D}^{\prime} \eta=\Delta_{\rho}^{\prime \prime} \eta=0$ and conversely. Thus $\eta$ is harmonic if either $\Delta_{D}^{\prime \prime} \eta=\Delta_{\rho}^{\prime} \eta=0$ or $\Delta_{D}^{\prime} \eta=\Delta_{\rho}^{\prime \prime} \eta=0$.

Put now

$$
\eta=\sum_{i_{1}<\cdots<i_{q}} \eta_{\bar{i}_{1} \cdots \bar{i}_{q}} \otimes\left(X_{i_{1}} \wedge \cdots \wedge X_{i_{q}}\right)
$$

Then by $(6.2)$

$$
\begin{aligned}
\left(\Delta_{\rho}^{\prime} \eta, \eta\right) & =\sum_{i_{1}<\cdots<i_{q}} \sum_{k} \int_{\Gamma \backslash G}\left(\rho\left(X_{\bar{k}}\right) \rho\left(X_{k}\right) \eta_{\bar{i}_{1} \cdots \bar{i}_{q}}(x), \eta_{\bar{i}_{1} \cdots \bar{i}_{q}}(x)\right)_{F} d v \\
& =\sum_{i_{1}<\cdots<i_{q}} \sum_{k} \int_{\Gamma \backslash G}\left(\rho\left(X_{k}\right) \eta_{\bar{i}_{1} \cdots \bar{i}_{q}}(x), \rho\left(X_{k}\right) \eta_{\bar{i}_{1} \cdots \bar{i}_{q}}(x)\right)_{F} d v .
\end{aligned}
$$

Therefore we have $\Delta_{\rho}^{\prime} \eta=0$ if and only if $\rho\left(X_{k}\right) \eta_{\bar{i}_{1} \cdots \bar{i}_{q}}(x)=0$ for all $x \in \Gamma \backslash G$, $k, i_{1}, \cdots, i_{q}=1, \cdots, N$. Analogously $\Delta_{D}^{\prime} \eta=0$ if and only if $X_{k} \eta_{\bar{i}_{1} \cdots \bar{i}_{q}}(x)=0$ for all $k, i_{1}, \cdots, i_{q}=1, \cdots, N$. Thus we get

Lemma 6. 1. Let $\eta$ be an element of $C^{0, q}\left(\mathfrak{g}^{C}, \mathfrak{l}^{C} ; \mathcal{F}^{1}\right)$. Then each of the following conditions is necessary and sufficient for $\eta$ to be harmonic:

1) $\Delta_{D}^{\prime \prime} \eta=0$ and

$$
\rho\left(X_{k}\right)\left(\eta_{\bar{i}_{1} \cdots \bar{i}_{q}}(x)\right)=0
$$

for all $x \in \Gamma \backslash G$ and $k, i_{1}, \cdots, i_{q}=1, \cdots, N$.

2) $\Delta_{\rho}^{\prime \prime} \eta=0$ and

$$
\left(X_{k} \eta_{\bar{i}_{1} \cdots \bar{i}_{q}}\right)(x)=0
$$

for all $x \in \Gamma \backslash G$ and $k, i_{1}, \cdots, i_{q}=1, \cdots, N$.

Here $\eta_{\vec{i}_{1} \cdots \bar{i}_{q}}$ are the components of $\eta$ which are $F$-valued $C^{\infty}$-functions on $\Gamma \backslash G$. 


\section{Isomorphism between the cohomology groups} $\boldsymbol{H}^{0, q}(\Gamma, \boldsymbol{X}, \boldsymbol{\rho})$ and $\boldsymbol{H}_{\alpha^{\prime \prime}}^{0, q}\left(\Gamma, \boldsymbol{X}, \boldsymbol{J}_{\rho_{1}}\right)$

Let $\tau$ be a holomorphic representation of the group $K^{C}$ in a complex vector space $S$ and let $J_{\tau}$ be the canonical automorphic factor of type $\tau$. We first construct a bigraded complex which is canonically isomorphic to the complex $A\left(\Gamma, X, J_{\tau}\right)$.

Put

$$
\mathscr{Y}=\mathscr{R} \otimes{ }_{C} S
$$

where $\mathcal{R}$ is the vector space of all complex valued $C^{\infty}$-functions on $\Gamma / G$. As in the case of the vector space $\mathcal{F}, \mathscr{S}$ has $\mathfrak{t}^{C}$-module structures $l, l_{1}$ defined respectively by

$$
\begin{aligned}
l(Y)(f \otimes u) & =Y f \otimes u+f \otimes \tau(Y) u, \\
l_{1}(Y)(f \otimes u) & =Y f \otimes u
\end{aligned}
$$

for all $Y \in \mathfrak{l}^{c}, f \in \mathscr{R}, u \in S$. We can also define the operator $l_{1}(X)$ for any $X \in \mathfrak{g}^{C}$ by the same formula as above.

Let $L^{r}\left(\mathrm{~g}^{C} ; \mathscr{P}\right)$ be the vector space of all $r$-linear alternating mappings of $\mathrm{g}^{C}$ into $\mathscr{S}$. For a form $\eta \in L^{r}\left(\mathrm{~g}^{C} ; \mathscr{S}\right)$ and $Y \in \mathfrak{l}^{C}$ we define $\mathscr{L}(Y) \eta \in$ $L^{r}\left(\mathfrak{g}^{C} ; \mathscr{P}\right)$ and $i(Y) \eta \in L^{r-1}\left(\mathfrak{g}^{C} ; \mathscr{Y}\right)$ by putting

$$
\begin{aligned}
& (\mathscr{L}(Y) \eta)\left(Z_{1}, \cdots, Z_{r}\right)=l(Y)\left(\eta\left(Z_{1}, \cdots, Z_{r}\right)\right)-\sum_{u=1}^{r} \eta\left(Z_{1}, \cdots,\left[Y, Z_{u}\right], \cdots, Z_{r}\right) \\
& (i(Y) \eta)\left(Z_{1}, \cdots, Z_{r-1}\right)=\eta\left(Y, Z_{1}, \cdots, Z_{r-1}\right)
\end{aligned}
$$

for $Z_{1}, \cdots, Z_{r} \in \mathrm{g}^{C}$. Let $L^{r}\left(\mathrm{~g}^{C}, \mathfrak{l}^{C} ; \mathscr{P}\right)$ be the subspace of $L^{r}\left(\mathfrak{g}^{C} ; \mathscr{Y}\right)$ consisting of all elements $\eta$ such that

$$
\left\{\begin{array}{c}
\mathscr{L}(Y) \eta=0, \\
i(Y) \eta=0
\end{array}\right.
$$

for all $Y \in \mathfrak{l}^{C}$. Just as in the case of $C^{r}\left(\mathfrak{g}^{C}, \mathfrak{l}^{C} ; \mathcal{F}\right)$ we define in $L^{r}\left(\mathfrak{g}^{C}, \mathfrak{l}^{C} ; \mathscr{P}\right)$ the subspace $L^{p, q}\left(\mathrm{~g}^{c}, \mathfrak{t}^{c} ; \mathscr{P}\right)$ of all elements of type $(p, q)(p+q=r)$. Then $L^{r}\left(\mathfrak{g}^{C}, \mathfrak{l}^{C} ; \mathscr{Y}\right)=\sum_{p^{+q=r}} L^{p, q}\left(\mathfrak{g}^{C}, \mathfrak{l}^{C} ; \mathscr{Y}\right)$. We may identify $L^{p, q}\left(\mathfrak{g}^{C}, \mathfrak{l}^{C} ; \mathscr{Y}\right)$ with the subspace of $\mathscr{S} \otimes \bigwedge^{p} \mathfrak{n}^{-} \otimes \bigwedge^{q} \mathfrak{n}^{+}$by the mapping

$$
i: \eta \rightarrow \sum_{i_{1}<\cdots<i_{p}} \sum_{j_{1}<\cdots j_{q}} \eta_{i_{1} \cdots i_{p}} \bar{j}_{1} \cdots \bar{j}_{q} \otimes \bigwedge_{s=1}^{p} X_{\bar{i}_{s}} \otimes \bigwedge_{t=1}^{q} X_{j_{t}}
$$

where $\eta_{i_{1} \cdots i_{p}} \bar{j}_{1} \cdots \bar{j}_{q}=\eta\left(X_{i_{1}}, \cdots, X_{i_{p}}, X_{\bar{j}_{1}}, \cdots, X_{\bar{j}_{q}}\right)$. Then $L^{p, q}\left(\mathfrak{g}^{C}, \mathfrak{l}^{C} ; \mathscr{\varphi}\right)$ consists of all $\eta \in \mathscr{S} \otimes \bigwedge^{p} \mathfrak{n}^{-} \otimes \bigwedge^{q} \mathfrak{n}^{+}$such that

$$
\left(l \otimes \operatorname{ad}_{-} \otimes \operatorname{ad}_{+}\right)(Y) \cdot \eta=0
$$

for all $Y \in \mathfrak{l}^{C}$, We can define a coboundary operator $d^{\prime \prime}$ of degree $(0,1)$ 
in the bigraded module $L\left(\mathfrak{g}^{C}, \mathfrak{f}^{C} ; \mathscr{Y}\right)=L^{p, q}\left(\mathrm{~g}^{C}, \mathfrak{l}^{C} ; \mathscr{Y}\right)$ by putting

$$
d^{\prime \prime}=(-1)^{p} \sum_{j=1}^{N} l_{1}\left(X_{\bar{k}}\right) \otimes 1 \otimes \varepsilon\left(X_{k}\right)
$$

on $L^{p, q}\left(\mathrm{~g}^{C}, \mathfrak{l}^{C} ; \mathscr{P}\right)$. Thus $L\left(\mathrm{~g}^{C}, \mathfrak{l}^{C} ; \mathscr{Y}\right)$ is a bigraded complex with coboundary operator $d^{\prime \prime}$.

There exists a canonical isomorphism of the bigraded complex $A\left(\Gamma, X, J_{\tau}\right)$ with the coboundary operator $d^{\prime \prime}$ onto the bigraded complex $L\left(\mathfrak{g}^{C}, \mathfrak{l}^{C} ; \mathscr{Y}\right)$; this is seen by comparing (1.4) with (7.1) and (1.5) with (7.2). Therefore we identify the group $H_{a^{\prime \prime}}^{p, q}\left(\Gamma, X, J_{\tau}\right)$ with the cohomology group of the complex $L\left(\mathrm{~g}^{C}, \mathfrak{l}^{C} ; \mathscr{P}\right)$. Each cohomology class in $H_{a^{\prime \prime}}^{p, q}\left(\Gamma, X, J_{\tau}\right)$ is represented by a unique harmonic element. Here we call an element $\eta$ of $L^{p, q}\left(\mathrm{~g}^{C}, \mathfrak{l}^{C} ; \varphi\right)$ harmonic, if $\eta$ is annihilated by the operator $\square$ " of type $(0,0)$ defined by

$$
\square^{\prime \prime}=-\sum_{k=1}^{N} l_{1}\left(X_{k}\right) l_{1}\left(X_{\bar{k}}\right) \otimes 1 \otimes 1-\sum_{a} l_{1}\left(Y_{a}\right) \otimes 1 \otimes \operatorname{ad}_{+}\left(Y_{a}\right) .
$$

(see [9, p. 403]).

Let now $\rho$ be an irreducible representation of $G^{C}$ in a complex vector space $F$. We denote by $\Lambda$ the highest weight of the representation $\rho$, and let $F_{\Lambda}$ be the (1-dimensional) eigen-space of the highest weight $\Lambda$.

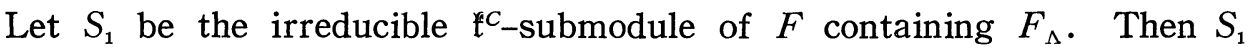
coincides with the subspace of $F$ consisting of all $u \in F$ such that $\rho(X) u$ $=0$ for all $X \in \mathfrak{n}^{+}\left[9\right.$, p. 409]. We take as representation $\tau$ of $K^{c}$ the representation $\rho_{1}$ of $K^{C}$ in $S_{1}$ induced by $\rho$. Let $S_{1}^{\perp}$ be the orthogonal complement of $S_{1}$ in $F$ (with respect to the positive definite hermitian product $\left.(,)_{F}\right)$. Then $\mathcal{F}=\mathscr{R} \otimes F$ decomposes into the direct sum of the $\mathfrak{l}^{C}$-submodules $\mathscr{S}_{1}=\mathscr{R} \otimes S_{1}$ and $\mathscr{S}_{1}^{\perp}=\mathscr{R} \otimes S_{1}^{\perp}$ and $\mathscr{Y}_{1}$ consists of all $f \in \mathcal{F}_{1}$ such that $m_{2}(X) f=0$ for all $X \in \mathfrak{n}^{+}$. The vector space $L^{r}\left(\mathfrak{g}^{c}, \mathfrak{l}^{C} ; \mathscr{\varphi}_{1}\right)$ is canonically identified with a subspace of $C^{r}\left(\mathfrak{g}^{C}, \mathfrak{l}^{C} ; F^{\prime}\right)$ and under this identification $L^{p, q}\left(\mathfrak{g}^{C}, \mathfrak{l}^{C} ; \mathscr{Y}_{1}\right)$ is identified with the subspace of $C^{p, q}\left(\mathfrak{g}^{C}, \mathfrak{l}^{C} ; \mathcal{F}\right)$ consisting of all elements $\eta$ such that $\left(m_{2}(X) \otimes 1 \otimes 1\right) \eta=0$ for all $X \in \mathfrak{n}^{+}$. Moreover, as we see from (5.1) and (7.3), the operator $\square^{\prime \prime}$ is the restriction of $\Delta_{D}^{\prime \prime}$ into $L^{p, q}\left(\mathfrak{g}^{C}, \mathfrak{l}^{C} ; \mathscr{Y}_{1}\right)$. Thus a harmonic element $\eta$ of $L^{p, q}\left(\mathfrak{g}^{C}, \mathfrak{l}^{C} ; \mathscr{Y}_{1}\right)$ is just an element $\eta$ of $C^{p, q}\left(\mathfrak{g}^{C}, \mathfrak{l}^{C} ; \mathcal{F}_{1}\right)$ such that $\Delta_{D}^{\prime \prime} \eta=0$ and $\left(m_{2}\left(X_{k}\right) \otimes 1 \otimes 1\right) \eta=0$ for $k=1, \cdots, N$. It follows from Lemma 6.1 that the space of harmonic elements in $L^{0, q}\left(\mathrm{~g}^{C}, \mathfrak{l}^{C} ; \mathscr{Y}_{1}\right)$ coincides with the space of harmonic cocycles in $C^{0, q}\left(\mathfrak{g}^{C}, \mathfrak{l}^{C} ; \mathcal{F}^{\prime}\right)$. From this we get the following theorem which is proved in [9] in the special case $q=N$.

Theorem 7.1. Let $\rho$ be an irreducible representation of $G^{C}$ in $a$ complex vector space $F$ and let $\rho_{1}$ be the irreducible representation of $K^{c}$ as defined above. Then the cohomology group $H_{a^{\prime \prime}}^{0, q}\left(\Gamma, X, J_{\rho_{1}}\right)$ is canonically 
isomorphic to the cohomology group $H^{0, q}(\Gamma, X, \rho)$ for $q=0, \cdots, N$.

We shall need later in $\S 9$ the following lemma, which follows from the well-known Serre's duality theorem in the case where $\Gamma$ acts freely on $X$.

Lemma 7.1. Let $\tau$ be a holomorphic representation of $K^{c}$ in a complex vector space $S$, and let ${ }^{{ }^{t} \tau^{-1}}$ be its contragradient representation of $K^{c}$ in the dual vector space $S^{*}$ of $S$. Let $\sigma$ be the 1-dimensional representation of $K^{c}$ defined by $\sigma(t)=\operatorname{det}\left(\operatorname{ad}_{-}^{1}(t)\right)$ for $t \in K^{c}$, where ad ${ }^{1}$ is the representation of $K^{c}$ in the $K^{c}$-module $\mathfrak{n}^{-}$. Then we have

$$
\operatorname{dim}_{C} H_{a^{\prime \prime}}^{0, q}\left(\Gamma, X, J_{\tau}\right)=\operatorname{dim}_{C} H_{a^{\prime \prime \prime}}^{0, N-q}\left(\Gamma, X, J_{\sigma \otimes t_{\tau}-1}\right)
$$

for $q=0, \cdots, N$.

Proof. It is sufficient to show that the vector space of harmonic elements in $L^{0, q}\left(\mathrm{~g}^{C}, \mathfrak{l}^{C} ; \mathscr{S}\right)$ and the vector space of harmonic elements in $L^{0, N-q}\left(\mathfrak{g}^{C}, \mathfrak{l}^{C} ; \varphi^{*}\right)$ are of same dimension, where $\varphi^{*}$ is the module $\mathscr{R} \otimes S^{*}$ with the $\mathfrak{l}^{C}$-module structure $l^{*}$ defined by

$$
l^{*}(Y)(f \otimes u)=Y f \otimes u+f \otimes\left(\left(\sigma \otimes{ }^{t} \tau^{-1}\right)(Y)\right) u
$$

for $Y \in \mathfrak{l}^{C}, f \in \mathscr{R}, u \in S^{*}$. The module $\mathscr{R} \otimes S^{*}$ has also two other $\mathfrak{l}^{C}$ module structures defined by

$$
\begin{aligned}
& l_{1}^{*}(Y)(f \otimes u)=Y f \otimes u, \\
& l_{2}^{*}(Y)(f \otimes u)=Y f \otimes u+f \otimes^{t} \tau^{-1}(Y) u .
\end{aligned}
$$

We can define $l_{1}^{*}(X)$ for any $X \in \mathrm{g}^{c}$ by the same formula.

We now introduce in $S$ a positive definite hermitian inner product $(,)_{S}$ invarinat under all $\tau(t)(t \in K)$. Then every element $u \in S$ defines an element $\sharp u$ of $S^{*}$ such that $(\sharp u)(v)=(v, u)$ for all $v \in S$. The mapping $u \rightarrow \# u$ is a conjugate linear bijection of $S$ onto $S^{*}$. We can then define a conjugate linear bijection, denoted also by $\#$, of $\mathscr{Y}=\mathscr{R} \otimes S$ onto $\mathscr{S}^{*}$ $=\mathscr{R} \otimes S^{*}$ such that $\#(f \otimes u)=\bar{f} \otimes(\# u)$ for $f \in \mathscr{R}$ and $u \in S$, where $\bar{f}$ is the complex conjugate of the function $f$. We verify then easily

$$
\left\{\begin{array}{l}
\sharp l_{1}(X)(f \otimes u)=l_{1}^{*}(\bar{X}) \#(f \otimes u), \\
\sharp l(Y)(f \otimes u)=l_{2}^{*}(\bar{Y}) \#(f \otimes u)
\end{array}\right.
$$

for $X \in \mathrm{g}^{C}, Y \in \mathfrak{l}^{C}$ and $(f \otimes u) \in \mathscr{P}$, where - denotes the conjugation of $\mathrm{g}^{C}$ with respect to $\mathfrak{g}$. On the other hand, we can define a conjugate linear bijection $\bar{*}$ of $\bigwedge^{q} \mathfrak{n}^{+}$onto $\stackrel{N}{\Lambda}^{-q} \mathfrak{n}^{+}$by putting $\bar{*}\left(X_{j_{1}} \wedge \cdots \wedge X_{j_{q}}\right)=\sum_{k_{1}<\cdots<k_{N-q}} \operatorname{sgn}\left(\begin{array}{l}1, \cdots \cdots \cdots \cdots \cdots, \cdots, N \\ j_{1}, \cdots, j_{q}, k_{1}, \cdots, k_{N-q}\end{array}\right)\left(X_{k_{1}} \wedge \cdots \wedge X_{N-q}\right)$. 
It is then not difficult to see that the following formula holds:

$$
\bar{*}\left(\operatorname{ad}_{+}(Y) v\right)=\left(\left(\sigma \otimes \operatorname{ad}_{+}\right)(\bar{Y})\right)(\bar{*} v)
$$

for any $Y \in \mathfrak{l}^{c}$ and $v \in \bigwedge^{a} \mathfrak{n}^{+}$. Now the mappings $\#$ and $\bar{*}$ define canonically a conjugate linear bijection $\# \otimes \bar{*}$ of $\varphi \otimes \bigwedge^{q} \mathfrak{n}^{+}$onto $\varphi^{*} \otimes \otimes^{N} \bigwedge^{-q} \mathfrak{n}^{+}$. From (7.4) and (7.5), it follows that this mapping $\# \otimes \bar{*}$ maps $L^{0, q}\left(\mathrm{~g}^{c}, \ell^{C} ; \varphi\right)$ onto $L^{0, N-q}\left(\mathfrak{g}^{C}, \mathfrak{f}^{C} ; \varphi^{*}\right)$; moreover, using (4.3), (4.4) and (7.3), we see that

$$
\square^{\prime \prime}((\# \otimes \bar{*}) \eta)=(\# \otimes \bar{*})\left(\square \square^{\prime \prime} \eta\right)
$$

holds for all $\eta \in L^{0, q}\left(\mathfrak{g}^{c}, \mathfrak{l}^{c} ; \varphi\right)$, where $\square^{\prime \prime}$ denotes the laplacian in $L^{0, N-q}\left(\mathfrak{g}^{C}, \mathfrak{l}^{C} ; \varphi^{*}\right)$ which is defined by a formula analogous to (7.3). Thus the mapping $\# \otimes \bar{*}: L^{0, q}\left(\mathrm{~g}^{C}, \mathfrak{p}^{C} ; \varphi\right) \rightarrow L^{0, N-q}\left(\mathrm{~g}^{C} ; \mathfrak{l}^{C} ; \varphi^{*}\right)$ defines a bijective conjugate linear mapping between harmonic elements, and this proves the Lemma.

\section{Vanishing theorems for the cohomology groups $\boldsymbol{H}^{0, q}(\Gamma, \boldsymbol{X}, \boldsymbol{\rho})$}

By restricting the representation $\rho$ of $\mathrm{g}^{c}$ onto the abelian subalgebra $\mathfrak{n}^{-}$, we may consider $F$ as an $\mathfrak{n}^{-}$-module. Let $C\left(\mathfrak{n}^{-}, F\right)=\sum_{q} C^{q}\left(\mathfrak{n}^{-}, F\right)$ be the cochain complex of the abelian Lie algebra $\mathfrak{n}^{-}$with coefficients in $F$, and denote by $d^{-}$its coboundary operator. We identify canonically the graded module $C\left(\mathfrak{n}^{-}, F\right)=\sum_{q} C^{q}\left(\mathfrak{n}^{-}, F\right)$ with $F \otimes \wedge \mathfrak{n}^{+}=\sum_{q} F \otimes \wedge \mathfrak{n}^{+}$. Then we have

$$
d^{-}=\sum_{k=1}^{N} \rho\left(X_{\bar{k}}\right) \otimes \varepsilon\left(X_{k}\right) .
$$

We define a positive definite hermitian inner product in $C\left(\mathfrak{n}^{-}, F\right)$ by

$$
\left(c, c^{\prime}\right)=\sum_{q} \sum_{i_{1}<\cdots<i_{q}}\left(c_{\bar{i}_{1} \cdots \bar{i}_{q}}, c^{\prime}{\overline{i_{1}} \cdots \bar{i}_{q}}_{q_{F}}\right)_{F},
$$

where $c=\sum_{q} \sum_{i_{1}<\cdots i_{q}} c_{\bar{i}_{1} \cdots \bar{i}_{q}} \otimes\left(X_{i_{1}} \wedge \cdots \wedge X_{i_{q}}\right)$ and $c^{\prime}=\sum_{q} \sum_{i_{1}<\cdots<i_{q}} c_{i_{1} \cdots i_{q}}^{\prime} \otimes$ $\left(X_{i_{1}} \wedge \cdots \wedge X_{i_{q}}\right)$.

Let $\delta^{-}$be the adjoint operator of $d^{-}$with respect to this inner product and we define an operator $\Delta^{-}$, referred to as a laplacian, by putting

$$
\Delta^{-}=d^{-} \delta^{-}+\delta^{-} d^{-} .
$$

We call a cocycle $c$ of $C\left(\mathfrak{n}^{-}, F\right)$ harmonic, if $\Delta^{-} c=0$. Every cohomology class in $H^{q}\left(\mathfrak{n}^{-}, F\right)$ is represented by one and only one harmonic cocycle (cf. [8]). By calculations analogous to those in [9], we find the following formulae : 


$$
\left\{\begin{aligned}
\delta^{-} & =\sum_{k=1}^{N} \rho\left(X_{k}\right) \otimes i\left(X_{\bar{k}}\right), \\
\Delta^{-} & =\sum_{k=1}^{N} \rho\left(X_{k}\right) \rho\left(X_{\bar{k}}\right) \otimes 1+\sum_{a} \rho\left(Y_{a}\right) \otimes \operatorname{ad}_{+}\left(Y_{a}\right) .
\end{aligned}\right.
$$

Now, for each point $x \in \Gamma \backslash G$, we define a mapping $\eta \rightarrow \eta(x)$ of $C^{0, q}\left(\mathfrak{g}^{C}, \mathfrak{l}^{C} ; \mathcal{F}^{\prime}\right)$ into $C\left(\mathfrak{n}^{-}, F\right)$ as follows. If

$$
\eta=\sum_{q} \sum_{i_{1}<\cdots<i_{q}} \eta_{\vec{i}_{1} \cdots \vec{i}_{q}} \otimes\left(X_{i_{1}} \wedge \cdots \wedge X_{i_{q}}\right),
$$

then

$$
\eta(x)=\sum_{q} \sum_{i_{1}<\cdots<i_{q}} \eta_{\bar{i}_{1} \cdots \bar{i}_{q}}(x) \otimes\left(X_{i_{1}} \wedge \cdots \wedge X_{i_{q}}\right) .
$$

It follows from (5.1) and (8.1) that

$$
\left(\Delta_{\rho}^{\prime \prime} \eta\right)(x)=\Delta^{-}(\eta(x))
$$

holds for all $x \in \Gamma \backslash G$. By Lemma 6.1 we get the following

Lemma 8. 1. If $\eta$ is a harmonic cocycle in $C^{0, q}\left(\mathfrak{g}^{C}, \mathfrak{l}^{C}\right.$; F $)$, then $\eta(x)$ is a harmonic cocycle in $C^{q}\left(\mathfrak{n}^{-}, F\right)$ such that $\rho\left(X_{k}\right)\left(\eta_{\bar{i}_{1} \cdots \bar{i}_{q}}(x)\right)=0$ for $k=1$, $\cdots, N, x$ being an arbitrary point of $\Gamma \backslash G$.

From the formula (8.1) we obtain the following formula for the laplacian $\Delta^{-}$:

$$
2 \Delta^{-}=\rho(C) \otimes 1+\sum_{a}\left(\rho \otimes \operatorname{ad}_{+}\right)\left(Y_{a}\right)^{2}+\sum_{k}\left(\rho \otimes \operatorname{ad}_{+}\right)\left(\left[X_{k}, X_{k}\right]\right)
$$

The proof of (8.2) is parallel to the proof of the formula (5.6) for $\Delta_{\rho}^{\prime \prime}$.

The module $F \otimes \wedge \mathfrak{n}^{+}$will be considered as a $\mathfrak{l}^{c}$-module by the representation $\rho \otimes \mathrm{ad}_{+}$of $\mathfrak{l}^{C}$ in $F \otimes \wedge \mathfrak{n}^{+}$. It follows from (8.2) that the $\mathfrak{l}^{C}$-submodule $S_{1} \otimes \bigwedge^{q} \mathfrak{n}^{+}$is stable under the operator $\Delta^{-}$and we denote by $\Delta_{q}^{-}$the restriction of $\Delta^{-}$into $S_{1} \otimes \bigwedge^{q} \mathfrak{n}^{+}$. Since $\rho\left(X_{k}\right) u=0$ for $u \in S_{1}$ and $k=1, \cdots, N$, we have $\rho\left(\left[X_{k}, X_{\bar{k}}\right]\right)=\rho\left(X_{k}\right) \rho\left(X_{\bar{k}}\right)+\rho\left(X_{\bar{k}}\right) \rho\left(X_{k}\right)$ on $S_{1}$ and hence we have

$$
\sum_{k} \rho\left(\left[X_{k}, X_{\bar{k}}\right]\right)=\rho(C)+\sum_{a} \rho\left(Y_{a}\right)^{2}
$$

on $S_{1}$. By this and Lemma 4.1 the last term of (8.2) is:

$$
\begin{aligned}
& \sum_{k}\left(\rho \otimes \operatorname{ad}_{+}\right)\left(\left[X_{k}, X_{k}\right]\right) \\
& \quad=\sum_{k} \rho\left(\left[X_{k}, X_{\bar{k}}\right]\right) \otimes 1+1 \otimes \sum_{k} \operatorname{ad}_{+}\left(\left[X_{k}, X_{\bar{k}}\right]\right) \\
& \quad=\rho(C) \otimes 1+\sum_{a} \rho\left(Y_{a}\right)^{2} \otimes 1-1 \otimes \sum_{a} \operatorname{ad}_{+}\left(Y_{a}\right)^{2} .
\end{aligned}
$$

on $S_{1} \otimes \bigwedge^{q} \mathfrak{n}^{+}$. We put

$$
C^{\prime}=-\Sigma_{a} Y_{a}^{2}
$$

$C^{\prime}$ is an element of the center of the envelopping algebra $\varepsilon^{\prime}$ of 
Then, extending the representations of $\mathfrak{f}^{C}$ to those of $\mathscr{E}^{\prime}$, we get from (8.2) the following formula:

$$
2 \Delta_{q}^{-}=2 \rho(C) \otimes 1-\rho\left(C^{\prime}\right) \otimes 1+1 \otimes \operatorname{ad}_{+}\left(C^{\prime}\right)-\left(\rho \otimes \operatorname{ad}_{+}\right)\left(C^{\prime}\right) .
$$

Now we choose an ordering of the roots such that the roots belonging to $\Psi$ are all positive. Let $\Theta$ be the set of all positive roots $\alpha$ of $\mathrm{g}^{C}$ such that $X_{a} \in \mathfrak{l}^{C}, X_{\alpha}$ being an eigenvector for the root $\alpha$. Then $\Sigma_{+}=\Psi \cup \Theta$ is the set of all positive roots. We denote by $\left\{\gamma_{1}, \cdots, \gamma_{l}\right\}$ the set of all simple roots and we may assume that $\gamma_{1}, \cdots, \gamma_{s}$ are in $\Psi$ and $\gamma_{s+1}, \cdots, \gamma_{l}$ are in $\Theta$. It is known that the number $s$ equals the number of simple factors of $\mathrm{g}^{c}$ and that $\gamma_{s+1}, \cdots, \gamma_{l}$ are also simple as roots of the complex reductive Lie algebra $\mathfrak{f}^{C}$. Under these notations, if $\rho$ (resp. $\tau$ ) is an irreducible representation of $\mathrm{g}^{C}$ (resp. $\mathfrak{l}^{C}$ ) with highest weight $\Lambda$ (resp. $\Lambda^{\prime}$ ), then $\rho(C)$ and $\tau\left(C^{\prime}\right)$ are scalar operators and we have

$$
\begin{aligned}
& \rho(C)=\left\{\langle\Lambda, \Lambda\rangle+\sum_{\alpha \in \Psi \cup \Theta}\langle\Lambda, \alpha\rangle\right\} 1, \\
& \tau\left(C^{\prime}\right)=\left\{\left\langle\Lambda^{\prime}, \Lambda^{\prime}\right\rangle+\sum_{\beta \in \Theta}\left\langle\Lambda^{\prime}, \beta\right\rangle\right\} 1 .
\end{aligned}
$$

Indeed, these formulae are easily proved by calculating the effect of $\rho(C)$ (resp. $\tau\left(C^{\prime}\right)$ ) to an eigenvector belonging to the highest weight $\Lambda$ (resp. $\Lambda^{\prime}$ ).

Theorem 8.1. Let $\rho$ be an irreducible representation of $G^{c}$ in a complex vector space $F$ and let $\Lambda$ be the highest weight of $\rho$. Let $c$ be a harmonic cocycle of $C^{q}\left(\mathfrak{n}^{-}, F\right)$ such that $\rho(X) c_{\bar{i} \cdots \bar{i}_{q}}=0$ for $i_{1}, \cdots, i_{q}=1, \cdots, N$ and for all $X \in \mathfrak{n}^{+}$, where $c_{\bar{i}_{1} \cdots \bar{i}_{q}}=c\left(X_{\bar{i}_{1}}, \cdots, X_{\bar{i}_{q}}\right)$. Let $q_{\rho}$ be the number of roots $\alpha$ belonging to $\Psi$ such that $\langle\Lambda, \alpha\rangle\rangle 0$. Then, if $q\left\langle q_{\rho}, c\right.$ is equal to 0 .

Proof. Let

$$
\bigwedge^{a} \mathfrak{n}^{+}=M_{1}+\cdots+M_{h}
$$

be a decomposition of the $\mathfrak{l}^{c}$-module $\bigwedge^{q} \mathfrak{n}^{+}$into direct sum of irreducible $\mathfrak{f}^{C}$-submodules and we denote by $\Lambda_{j}$ the highest weight of the irreducible representation $M_{j}^{c}$ in $(j=1, \cdots, h)$. Then each $\Lambda_{j}$ is a sum of $q$ roots in $\Psi$. Let further

$$
S_{1} \otimes M_{j}=U_{j_{1}}+\cdots+U_{j_{j}}
$$

be a decomposition of the $\mathfrak{l}^{C}$-module $S_{1} \otimes M_{j}$ into direct sum of irreducible $\mathfrak{l}^{C}$-submodules. If we denote by $\Lambda$ the highest weight of the representation $\rho$ of $g^{C}$ in $F$, the highest weight of the representation of $\mathfrak{l}^{C}$ in 
$S_{1} \otimes M_{j}$ is just equal to $\Lambda+\Lambda_{j}$ and we may arrange the numbering of $U_{j_{1}}$ in the way that the highest weight $\xi_{j_{1}}$ of the irreducible representation of $\mathfrak{i n}^{c}$ in $U_{j_{1}}$ is $\Lambda+\Lambda_{j}$. Let $\xi_{j i}$ be the highest weight of the irreducible representation of $\mathfrak{t}^{C}$ in $U_{j i}$. Then we have

$$
\left\{\begin{array}{l}
\xi_{j_{1}}=\Lambda+\Lambda_{j}, \\
\xi_{j i}=\xi_{j_{1}}-\sum_{t=s+1}^{l} m_{t} \gamma_{t} \quad(i>1)
\end{array}\right.
$$

where $m_{t}(t=s+1, \cdots, l)$ are non-negative integers.

It follows from the formula (8.3) that the laplacian $\Delta_{q}^{-}$leaves invariant the subspaces $U_{j i}$ of $F \otimes \bigwedge^{q} \mathfrak{n}^{-}$and is a scalar operator $a_{j i}^{(q)} 1$ on each $U_{j i}$. In view of (8.3), (8.4) and (8.5), the scalar $a_{j i}^{(q)}$ is given by

$$
\begin{aligned}
2 a_{j i}^{(q)}= & 2\left(\langle\Lambda, \Lambda\rangle+\sum_{\alpha \in \Psi \cup \Theta}\langle\Lambda, \alpha\rangle\right)-\left(\langle\Lambda, \Lambda\rangle+\sum_{\beta \in \Theta}\langle\Lambda, \beta\rangle\right) \\
& +\left(\left\langle\Lambda_{j}, \Lambda_{j}\right\rangle+\sum_{\beta \in \Theta}\left\langle\Lambda_{j}, \beta\right\rangle\right)-\left(\left\langle\xi_{j i}+\xi_{j i}\right\rangle+\sum_{\beta \in \Theta}\left\langle\xi_{j i}, \beta\right\rangle\right) .
\end{aligned}
$$

Now we get from (8.6)

$$
\begin{aligned}
& \left\langle\xi_{j_{1}}, \xi_{j_{1}}\right\rangle=\left\langle\xi_{j i}+\sum_{t=s+1}^{l} m_{t} \gamma_{t}, \xi_{j i}+\sum_{t=s+1}^{l} m_{t} \gamma_{t}\right\rangle \\
& \quad=\left\langle\xi_{j i}, \xi_{j i}\right\rangle+2 \sum_{t=s+1}^{l} m_{t}\left\langle\xi_{j i}, \gamma_{t}\right\rangle+\left\langle\sum_{t=s+1}^{l} m_{t} \gamma_{t}, \sum_{t=s+1}^{l} m_{t} \gamma_{t}\right\rangle .
\end{aligned}
$$

Since $\xi_{j i}$ is the highest weight of an irreducible representation of $\mathfrak{l}^{C}$ and $\gamma_{t} \in \Theta$, we have $\left\langle\xi_{j i}, \gamma_{t}\right\rangle \geqq 0$, and hence we get

$$
\left.\left\langle\xi_{j 1}, \xi_{j_{1}}\right\rangle\right\rangle\left\langle\xi_{j i}, \xi_{j i}\right\rangle \text {. }
$$

Moreover, we have

$$
\sum_{\beta \in \Theta}\left\langle\xi_{j_{1}}, \beta\right\rangle=\sum_{\beta \in \Theta}\left\langle\xi_{j i}, \beta\right\rangle+\sum_{t=s+1}^{l} m_{t}\left\langle\gamma_{t}, \sum_{\beta \in \Theta} \beta\right\rangle .
$$

Since $s^{\prime}=\sum_{\beta \in \Theta} \beta$ is the sum of positive roots of $\left[\mathfrak{t}^{c}, \mathfrak{l}^{c}\right]$, we have $\left\langle\gamma, s^{\prime}\right\rangle>0$ for all $\gamma \in \Theta$, and hence we get

$$
\sum_{\beta \in \Theta}\left\langle\xi_{j_{1}}, \beta\right\rangle>\sum_{\beta \in \Theta}\left\langle\xi_{j i}, \beta\right\rangle .
$$

Therefore from (8.7) follows the inequality

$$
a_{j i}^{(q)}>a_{j 1}^{(q)} \quad \text { for } \quad i=2, \cdots, v_{j} .
$$

Since we have $\xi_{j_{1}}=\Lambda+\Lambda_{j}$, we get immediately from (8.7):

$$
a_{j 1}^{(q)}=\left\langle\Lambda, \sum_{\alpha \in \mathbb{\Psi}} \alpha-\Lambda_{j}\right\rangle .
$$

As $\Lambda_{j}$ is a sum of $q$ roots in $\Psi, \sum_{\alpha \in \Psi} \alpha-\Lambda_{j}$ is a sum of $N-q$ roots in $\Psi=\left\{\alpha_{1}, \cdots, \alpha_{N}\right\}$. Since $\Lambda$ is the highest weight of the irreducible representation of $\rho$, we have $\langle\Lambda, \alpha\rangle \geqq 0$ for a positive root $\alpha$ and hence $a_{j 1}^{(q)}$ is non-negative. It follow also that $a_{j 1}^{(q)}$ is positive for $j=1, \cdots, h$ if 
$q\left\langle q_{\rho}, q_{\rho}\right.$ being the number of roots belonging to $\Psi$ such that $\langle\Lambda, \alpha\rangle>0$.

If $a_{j_{1}}^{(q)}$ is positive for $j=1, \cdots, h$, we see from (8.7) and (8.8) that

the laplacian $\Delta_{q}^{-}$has no eigenvalue equal to 0 on $S_{1} \otimes \bigwedge^{q} \mathfrak{n}^{+}$and hence there is no harmonic cocycle different from 0 in $C^{q}\left(\mathfrak{n}^{-}, F\right)$ belonging to $S_{1} \otimes \bigwedge^{q} \mathfrak{n}^{+}$. This means that, if $a_{j 1}^{(q)}>0$ for $j=1, \cdots, h$ and if $c$ is a harmonic cocycle in $C^{q}\left(\mathfrak{n}^{-}, F\right)$ such that $\rho\left(X_{k}\right) c_{\bar{i}_{1} \cdots \bar{i}_{q}}=0$ for $k, i_{1}, \cdots, i_{q}=1, \cdots, N$, then $c$ is equal to 0 . As we have observed above, if $q<q_{\rho}$ we get $a_{j 1}^{(q)}>0$ for $j=1, \cdots, N$ and this proves the theorem.

REMARK. We shall see later in $\S 11$ that Theorem 8.1 is a consequence of a more general result which depends on a deep result of Kostant [8]. The present proof of Theorem 8.1 is more elementary. According to Raghunathan [11], the number $q_{\rho}$ in Theorem 8.1 is greater than 1 for almost all $\rho$ in the case where $G$ is simple.

From this theorem and Lemma 8.1, we get immediately the following theorem.

Theorem 8.2. The notation being as in Theorem 8.1, the cohomology group $H^{0, q}(\Gamma, X, \rho)$ vanishes for all $q<q_{\rho}$.

From this theorem follows:

Corollary 1. The notation being as in Theorem 8.1, suppose that $\left\langle\Lambda, \gamma_{i}\right\rangle>0$ for $i=1, \cdots, s$, where $\gamma_{1}, \cdots, \gamma_{s}$ are the simple roots belonging to $\Psi$. Then the cohomology group $H^{0, q}(\Gamma, X, \rho)$ vanishes for $q<N, N$ being the complex dimension of the symmetric bounded domain $X$.

Proof. We know that a root $\alpha \in \Psi$ is written in the form $\alpha=\gamma_{i}$ $+\sum_{t=s+1}^{l} m_{t} \gamma_{t}, 1 \leqq i \leqq s$, where $m_{t}(s+1 \leqq t \leqq l)$ are non-negative integers. Then $\langle\Lambda, \alpha\rangle=\left\langle\Lambda, \gamma_{i}\right\rangle+\sum_{t=s+1}^{l} m_{t}\left\langle\Lambda, \gamma_{t}\right\rangle$ and $\left\langle\Lambda, \gamma_{j}\right\rangle \geqq 0$ for $j=1, \cdots, l$. Therefore, if $\left\langle\Lambda, \gamma_{i}\right\rangle>0$ for $i=1, \cdots, s$, we have $\langle\Lambda, \alpha\rangle>0$ for all $\alpha \in \Psi$ and hence $q_{p}=N$. The corollary follows thus from Theorem 8.2.

Corollary 2. Let $\mathrm{g}^{c}$ be simple and let $\gamma_{1}$ be the (unique) simple root of $\mathrm{g}^{c}$ belonging to $\Psi$. Then, the cohomology group $H^{0, q}(\Gamma, X$, ad) vanishes for $q<\frac{1}{\left\langle\gamma_{1}, \gamma_{1}\right\rangle}-1$.

Proof. This follows from Theorem 8.2, if we show that $q_{\text {ad }}=$ $\frac{1}{\left\langle\gamma_{1}, \gamma_{1}\right\rangle}-1$. The highest weight of the adjoint representation ad of $\mathrm{g}^{c}$ is nothing but the highest root of $\mathrm{g}^{C}$, which we shall denote by $\beta_{0}$. We know that $\beta_{0}$ belongs to $\Psi$. Since $g^{C}$ is simple, we know also that the representation $\mathrm{ad}_{+}^{1}$ of $\mathfrak{l}^{C}$ in $\mathfrak{n}^{+}$is irreducible. The weights of this 
representation $\mathrm{ad}_{+}^{1}$ are the roots belonging to $\Psi$; the highest weight is $\beta_{0}$ and the lowest one is the unique simple root $\gamma_{1}$ belonging to $\Psi$. Therefore, there exists an element $R_{1}$ belonging to the Weyl group of $\mathfrak{l}^{C}$ which transforms the roots belonging to $\Psi$ among themselves and which maps $\beta_{0}$ to $\gamma_{1}$. Then, $\left\langle\beta_{0}, \alpha\right\rangle=\left\langle\gamma_{1}, R_{1}(\alpha)\right\rangle$ for $\alpha \in \Psi$, and hence the number $q_{\text {ad }}$ equals the number of roots $\alpha \in \Psi$ such that $\left\langle\gamma_{1}, \alpha\right\rangle>0$.

Now, if $\alpha \in \Psi$ and $\alpha \neq \gamma_{1}$, neither $\alpha+\gamma_{1}$ nor $\alpha-2 \gamma_{1}$ can be a root of $\mathrm{g}^{c}$. It follows that the number $\frac{2\left\langle\alpha, \gamma_{1}\right\rangle}{\left\langle\gamma_{1}, \gamma_{1}\right\rangle}$ is equal to 0 or 1 . On the other hand, we know by Lemma 4.2 that $2\left\langle\gamma_{1}, s\right\rangle=1$ where $s=\sum_{\alpha \in \Psi} \alpha$. By these we get

$$
\begin{aligned}
q_{\text {ad }} & =\sum_{\omega \in \Psi} \frac{2\left\langle\alpha, \gamma_{1}\right\rangle}{\left\langle\gamma_{1}, \gamma_{1}\right\rangle}-1 \\
& =\frac{2\left\langle s, \gamma_{1}\right\rangle}{\left\langle\gamma_{1}, \gamma_{1}\right\rangle}-1=\frac{1}{\left\langle\gamma_{1}, \gamma_{1}\right\rangle}-1,
\end{aligned}
$$

which proves the Corollary.

\section{Vanishing theorems for the cohomology groups $\boldsymbol{H}_{\boldsymbol{a}^{\prime \prime}}^{0, q}\left(\Gamma, \boldsymbol{X}, \boldsymbol{J}_{\tau}\right)$}

The following theorem follows immediately from Theorems 7.1 and 8. 2 and Corollary 1 of Theorem 8.2.

Theorem 9.1. Let $\rho$ be an irreducible representation of $G^{C}$ in a complex vector space $F$ and let $\Lambda$ be the highest weight of $\rho$. Let further $\rho_{1}$ be the irreducible representation of $K^{c}$ in the complex vector space $S_{1}, S_{1}$ being the irreducible $K^{C_{-}}$submodule of $F$ containing the eigenspace of the highest weight $\Lambda$. Then the cohomology group $H_{a^{\prime \prime \prime}}^{0, q}\left(\Gamma, X, J_{\rho_{1}}\right)$ vanishes for $q<q_{\rho}$, where $q_{\rho}$ is the number of roots $\alpha \in \Psi$ such that $\langle\Lambda, \alpha\rangle>0$.

If $\left\langle\Lambda, \gamma_{i}\right\rangle>0$ for $i=1, \cdots, s$, where $\gamma_{1}, \cdots, \gamma_{s}$ are the simple roots belonging to $\Psi$, then

$$
H_{a^{\prime \prime \prime}}^{0, q}\left(\Gamma, X, J_{\rho_{1}}\right)=(0)
$$

for $q<N$.

In the case where $\mathrm{g}^{C}$ is simple and $\rho$ is the adjoint representation ad of $\mathrm{g}^{C}, \rho_{1}$ is the representation $\operatorname{ad}_{+}^{1}$ of $K^{C}$ in $\mathfrak{n}^{+}$. Moreover, if $\Gamma$ operates freely on $X$, the group $H_{a^{\prime \prime \prime}}^{0, q}\left(\Gamma, X, J_{\mathrm{ad}_{+}^{1}}^{1}\right)$ is isomorphic to the $q$-dimensional cohomology group $H^{q}(\Gamma \backslash X, \Theta)$ of the complex manifold $\Gamma \backslash X$ with coefficients in the sheaf $\Theta$ of germs of holomorphic sections of the tangent bundle of $\Gamma \backslash X$ (cf. [9, p. 414]). Thus we get from Corollary 2 of Theorem 8.2 the following 
Corollary (Calabi-Vesentini). The notations being as above, the cohomology group $H^{q}(\Gamma \backslash X, \Theta)$ vanishes for $q<\frac{1}{\left\langle\gamma_{1}, \gamma_{1}\right\rangle}-1$.

REMARK. The theorem of Calabi-Vesentini [3] states that the group $H^{q}(\Gamma \backslash X, \Theta)$ vanishes for $q<\gamma(D)-1$; the number $\gamma(D)$ is equal to $R / N \lambda_{1}$, where $N=\operatorname{dim}_{C} X, R$ is the (constant) scalar riemannian curvature of $X$ and $\lambda_{1}$ is the smallest eigenvalue of a certain linear operator $Q$ defined in terms of the curvature tensor of $X$. It should be noted that $\gamma(D)$ is just equal to $\frac{1}{\left\langle\gamma_{1}, \gamma_{1}\right\rangle}$. Denoting by $R_{i j k h}$ the real components of the curvature tensor with respect to or thogonal frames, we have $R=\sum_{i, j=1}^{2 N} R_{i j i j}$ $=-\sum_{j=1}^{2 N} \delta_{i j} / 2=-\left(\operatorname{dim}_{R} X\right) / 2=-\operatorname{dim}_{C} X=-N$, while Borel [1] has proved that the number $\lambda_{1}$ is given by $-\left\langle\gamma_{1}, \gamma_{1}\right\rangle$. Thus we get $\gamma(D)=\frac{1}{\left\langle\gamma_{1}, \gamma_{1}\right\rangle}$. We remark also that the number $q_{\text {ad }}$ is equal to $m(G / K)+1$, where $m(G / K)$ is the number of roots $\alpha \in \Psi, \alpha \neq \gamma_{1}$, for which $\alpha-\gamma_{1}$ is a root of $\mathrm{g}^{C}$. This is seen from the first equality in (8.8) and this formula is used in [1] to compute $q_{\text {ad }}$.

Theorem 9.2. Suppose that $\mathrm{g}^{c}$ is simple. Let $\tau$ be a holomorphic irreducible representation of $K^{c}$ in a complex vector space $S$ and let $\Lambda$ be the highest weight of $\tau$. We denote by $\sigma$ the 1-dimensional representation of $K^{c}$ defined by $\sigma(t)=\operatorname{det}\left(\operatorname{ad}_{-}^{1}(t)\right)$ for $t \in K^{c}$, by $\gamma_{1}$ the unique simple root belonging to $\Psi$ and by $\beta_{0}$ the highest root of $\mathrm{g}^{C}$. Then

1) If $\left\langle\Lambda, \gamma_{1}\right\rangle>0$, then $H_{a^{\prime \prime \prime}}^{0, q}\left(\Gamma, X, J_{\tau}\right)=(0)$ for $q<N$.

2) Let $\sigma^{r} \otimes \tau$ be the representation of $K^{C}$ in $S$ defined by $\left(\sigma^{r} \otimes \tau\right)(s)$ $=\sigma(s)^{r} \tau(s)$ for $s \in K^{c}, r$ being an integer. If $r>-2\left\langle\Lambda, \gamma_{1}\right\rangle$, then $H_{a^{\prime \prime \prime}}^{0, q}\left(\Gamma, X, J_{\sigma^{-r} \otimes \tau}\right)=(0)$ for $q<N$.

3) If $\left\langle\Lambda, \beta_{0}\right\rangle<-\frac{1}{2}$, then $H_{a^{\prime \prime}}^{0, q}\left(\Gamma, X, J_{\tau}\right)=(0)$ for $q>0$.

Proof. Let $H^{C}$ be the Cartan subgroup of $G^{C}$ which corresponds to the Cartan subalgebra $\mathfrak{h}^{C}$ of $\mathrm{g}^{c}$. Then $H^{c}$ is clearly contained in $K^{c}$. We know that the kernel of the homomorphism $h \rightarrow \exp 2 \pi \sqrt{-1} h$ of $\mathfrak{h}^{c}$ onto $H^{c}$ contains the lattice of $\mathfrak{h}^{c}$ generated by $\left\{\frac{2 \alpha}{\langle\alpha, \alpha\rangle} ; \alpha \in \Sigma\right\}$. Since $\Lambda$ is a weight of the representation $\tau$ of the group $K^{c}$, it follows that the numbers $\frac{2\langle\Lambda, \alpha\rangle}{\langle\alpha, \alpha\rangle}$ are integers for all roots $\alpha$ of $\mathfrak{g}^{c}$. Let $\gamma_{1}$, $\cdots, \gamma_{l}$ be the simple roots of $g^{c}$; then $\gamma_{1} \in \Psi$ and $\gamma_{2}, \cdots, \gamma_{l}$ are simple roots of the reductive Lie algebra $\mathfrak{l}^{C}$. Thus, if we put $m_{i}=\frac{2\left\langle\Lambda, \gamma_{i}\right\rangle}{\left\langle\gamma_{i}, \gamma_{i}\right\rangle}$ 
for $i=1,2, \cdots, l$, then $m_{1}$ is an integer and $m_{2}, \cdots, m_{l}$ are non-negative integers. Let $\Lambda_{1}, \cdots, \Lambda_{l}$ be the fundamental highest weights of $g^{c}$, that is, $\Lambda_{i}$ is the weight such that $\frac{2\left\langle\Lambda_{i}, \gamma_{j}\right\rangle}{\left\langle\gamma_{j}, \gamma_{j}\right\rangle}=\delta_{i j}$ for $j=1, \cdots, l$. We have then $\Lambda=m_{1} \Lambda_{1}+\cdots+m_{l} \Lambda_{l}$. We shall now prove the three assertions 1 ), 2) and 3$)$.

1) The condition $\left.\left\langle\Lambda, \gamma_{1}\right\rangle\right\rangle 0$ is equivalent to $m_{1}>0$. In this case $\Lambda$ is a highest weight of $\mathrm{g}^{c}$, and hence there exists an irreducible representation $\rho$ of $\mathrm{g}^{C}$ in a complex vector space $F$ with highest weight $\Lambda$. The representation $\tau$ of $\mathfrak{t}^{c}$ is then equivalent to the representation $\rho_{1}$ of $\mathfrak{t}^{C}$ induced by $\rho$ in the complex vector space $S_{1}$, where $S_{1}$ is the irreducible $\mathfrak{f}^{c}$-submodule of $F$ containing the eigenspace of the weight $\Lambda$. We see then by Theorem 9.1 that $H_{a^{\prime \prime}}^{0, q}(\Gamma, X, \rho)=(0)$ for $q>N$. (Note that the representation $\rho$ need not be induced by a representation of the group $G^{c}$, since the proof of Theorem 9.1 is purely infinitesimal).

2) Since $\mathfrak{n}^{-}$is spanned by $\left\{X_{\bar{\alpha}} ; \alpha \in \Psi\right\}$, the weight of the 1-dimensional representation $\sigma$ of $K^{c}$ is given by $-s$ where $s=\sum_{\alpha \in \Psi} \alpha$. Since $s$ is in the center of $\mathfrak{t}^{c}$, we have $\left\langle s, \gamma_{j}\right\rangle=0$ for $i=2, \cdots, l$. On the other hand, we have $\frac{2\left\langle s, \gamma_{1}\right\rangle}{\left\langle\gamma_{1}, \gamma_{1}\right\rangle}=\frac{1}{\left\langle\gamma_{1}, \gamma_{1}\right\rangle}$ by Lemma 4.2, which shows that $s=\frac{1}{\left\langle\gamma_{1}, \gamma_{1}\right\rangle} \Lambda_{1}$. Now the highest weight of $\sigma^{-r} \otimes \tau$ is $(-r)(-s)+\Lambda=$ $\frac{r}{\left\langle\gamma_{1}, \gamma_{1}\right\rangle} \Lambda_{1}+\Lambda$ and we have $\left\langle r s+\Lambda, \gamma_{1}\right\rangle=\frac{r}{2}+\left\langle\Lambda, \gamma_{1}\right\rangle$, which is positive for $r\rangle-2\left\langle\Lambda, \gamma_{1}\right\rangle$. Thus the assertion 2) follows by applying the assertion 1)

3) By Lemma 7.1 we have

$$
\operatorname{dim}_{C} H_{d^{\prime \prime}}^{0, q}\left(\Gamma, X, J_{\tau}\right)=\operatorname{dim}_{C} H_{\sigma^{\prime \prime}}^{0, N-q}\left(\Gamma, X, J_{\sigma \otimes_{\tau^{-1}}}\right) .
$$

where ${ }^{t} \tau^{-1}$ is the representation of $K^{C}$ contragradient to $\tau$. We see that the representation ${ }^{t_{\tau^{-1}}}$ has $-\Lambda$ as its lowest weight. Now, since both $\left\{\gamma_{2}, \cdots, \gamma_{l}\right\}$ and $\left\{-\gamma_{2}, \cdots,-\gamma_{l}\right\}$ are fundamental systems of roots of the reductive Lie algebra $\mathfrak{k}^{c}$, there exists an element $R_{1}$ of the Weyl group of ${ }^{c}$ such that $R_{1}$ transforms the set $\left\{\gamma_{2}, \cdots, \gamma_{l}\right\}$ onto the set $\left\{-\gamma_{1}, \cdots\right.$, $\left.-\gamma_{l}\right\}$. Then $\left\langle R_{1}(-\Lambda), \gamma_{j}\right\rangle=\left\langle-\Lambda, R_{1}^{-1}\left(\gamma_{j}\right)\right\rangle \geqq 0$ for $j=2, \cdots, l$, which shows that $R_{1}(-\Lambda)$ is the highest weight of ${ }^{t} \tau^{-1}$. Consider the irreducible representation $\operatorname{ad}_{+}^{1}$ of $K^{C}$ in $\mathfrak{n}^{+}$instead of $\tau$. Then the highest weight of $\operatorname{ad}_{+}^{1}$ is the highest root $\beta_{0}$ while the representation ${ }^{t}\left(\operatorname{ad}_{+}^{1}\right)^{-1}$ is equivalent to the representation ad- whose highest weight is the root $-\gamma_{1}$. Therefore, we see that $R_{1}\left(-\beta_{0}\right)=-\gamma_{1}$ and so that $R_{1}\left(\beta_{0}\right)=\gamma_{1}$. Moreover, if we consider $R_{1}$ as an element of the Weyl group of $\mathrm{g}^{c}$, then $R_{1}$ 
transforms the roots belonging to $\Psi$ among themselves. It follows in particular that the highest weight $-s$ of $\sigma$ is left fixed by $R_{1}$. From these we see that the highest weight of $\sigma \otimes^{t} \tau^{-1}$ is $-s+R_{1}(-\Lambda)$ and

$$
\begin{gathered}
\left\langle-s+R_{1}(-\Lambda), \gamma_{1}\right\rangle=\left\langle-R_{1}^{-1}(s)-\Lambda, R_{1}^{-1}\left(\gamma_{1}\right)\right\rangle \\
=\left\langle-s-\Lambda, \beta_{0}\right\rangle=-\left(\left\langle s, \beta_{0}\right\rangle+\left\langle\Lambda, \beta_{0}\right\rangle\right) .
\end{gathered}
$$

By the assertion 1) and (9.1), the group $H^{0, q}\left(\Gamma, X, J_{\tau}\right)$ vanishes for $q>0$ if $\left.\left\langle-s+R_{1}(-\Lambda), \bar{\gamma}_{1}\right\rangle\right\rangle 0$, that is, if $\left\langle\Lambda, \beta_{0}\right\rangle\left\langle-\left\langle s, \beta_{0}\right\rangle\right.$. Since $\left\langle s, \beta_{0}\right\rangle=\frac{1}{2}$ by Lemma 4.2 , we conclude that $H^{0, q}\left(\Gamma, X, J_{\tau}\right)=(0)$ for $q>0$ if $\left\langle\Lambda, \beta_{0}\right\rangle$ $<-\frac{1}{2}$. Thus the theorem is completely proved.

EXAMPLE. We consider the case where $X$ is the symmetric bounded domain of type III. In this case we have $G^{C}=\operatorname{Sp}(n, C), K^{c}=\mathrm{GL}(n, C)$ and, for a representation $\tau$ of $K^{c}, J_{\tau}(s, x)$ is of the form $\tau\left({ }^{t}(c x+d)^{-1}\right)$ $\left[9\right.$, p. 397 and p. 414]. A Cartan subalgebra $\mathfrak{h}^{C}$ of $\mathfrak{g}^{C}$ such that $\mathfrak{h} \subset \mathfrak{f}$ is parametrized by $\left(\lambda_{1}, \cdots, \lambda_{n}\right)$ with $\left\langle\lambda_{i}, \lambda_{j}\right\rangle=\delta_{i j} /(4 n+4)$. We may put $\gamma_{1}=2 \lambda_{n}, \gamma_{i}=\lambda_{i-1}+\lambda_{i}(i=2, \cdots, n)$ and $\beta_{0}=2 \lambda_{1}$. The highest weight of an irreducible representation $\tau$ of $\mathfrak{l}^{C}=\mathfrak{g l}(n, C)$ is of the form $m_{1} \lambda_{1}+\cdots+m_{n} \lambda_{n}$ with the integral coefficients $m_{i}$ with $m_{1} \geqq m_{2} \geqq \cdots \geqq m_{n}$. The conditions in Theorem 9.2 are as follows: The condition in 1) means $m_{n}>0$, and the condition in 3) means $m_{1}<-(n+1)$. As for the condition in 2), we note that the representation $\sigma$ is given by $\sigma(t)=$ $(\operatorname{det} t)^{-(n+1)}$ for $t \in K^{C}=\mathrm{GL}(n, C)$ and so $J_{\sigma^{-r} \otimes_{T}}=\operatorname{det}(c x+d)^{-r(n+1)} \tau\left({ }^{t}(c x\right.$ $\left.+d)^{-1}\right)$. Since $2\left\langle\Lambda, \gamma_{1}\right\rangle=\frac{m_{n}}{n+1}$ the condition in 2) means $r>-\frac{m_{n}}{n+1}$.

\section{Cohomology of the abelian Lie algebras $\mathfrak{n}^{ \pm}$and the decompositions of the $\mathfrak{l}^{C}$-modules $F \otimes \wedge \mathfrak{n}^{ \pm}$}

In the sections 10,11 and 12 we shall study the cohomology groups $H^{p, q}(\Gamma, X, \rho)$. We assume always that $\rho$ is irreducible. We begin with the study of the cohomology groups $H\left(\mathfrak{n}^{ \pm}, F\right)$ of the abelian Lie algebras $\mathfrak{n}^{ \pm}$by utilizing the laplacians $\Delta^{ \pm}$as in $\S 8$ and we shall apply the results to the study of the cohomology groups $H^{p, q}(\Gamma, X, \rho)$. We shall need some results of Kostant and in this section we follow the work of Kostant [8] (see also Cartier [4]).

Let

$$
F \otimes \wedge \mathfrak{n}^{+}=\sum_{\xi^{\prime}} U_{\xi^{\prime}}
$$

be a decomposition of the $\mathfrak{l}^{C}$-module $F \otimes \wedge \mathfrak{n}^{+}$into direct sum of irre- 
ducible $\mathfrak{f}^{C}$-modules $U_{\xi^{\prime}}$, where $\xi^{\prime}$ denotes the lowest weight of the irreducible representation $\tau_{\xi^{\prime}}$ of $\mathfrak{k}^{c}$ in $U_{\xi^{\prime}}$. Now, since $s=\sum_{k}\left[X_{k}, X_{\bar{k}}\right]$ by Lemma 4.2, the formula (8.2) may be written in the form

$$
2 \Delta^{-}=\rho(C) \otimes 1-\left(\rho \otimes \mathrm{ad}_{+}\right)\left(C^{\prime}\right)+\left(\rho \otimes \mathrm{ad}_{+}\right)(s) .
$$

The element $s=\sum_{\infty \in \Phi} \alpha$ being in the center of $\mathfrak{l}^{c}$, the last term is a scalar operator in each $U_{\xi^{\prime}}$. Thus the laplacian $\Delta^{-}$is a scalar operator $a_{\xi^{\prime}} 1$ on $U_{\xi^{\prime}}$ and, by formulae analogous to (8.4) and (8.5), the scalar $a_{\xi^{\prime}}$ is given by

$$
2 a_{\xi^{\prime}}=\left(\left\langle\Lambda^{\prime}, \Lambda^{\prime}\right\rangle-2\left\langle\Lambda^{\prime}, \delta\right\rangle\right)-\left(\left\langle\xi^{\prime}, \xi^{\prime}\right\rangle-\left\langle\xi^{\prime}, s^{\prime}\right\rangle\right)+\left\langle\xi^{\prime}, s\right\rangle .
$$

Here $\Lambda^{\prime}$ denotes the lowest weight of the irreducible representation $\rho$ and we put

$$
\begin{aligned}
& \delta=\frac{1}{2} \sum_{\alpha \in \Sigma_{+}} \alpha \\
& s=\sum_{\omega \in \Phi} \alpha, \quad s^{\prime}=\sum_{\beta \in \Theta} \beta .
\end{aligned}
$$

Since $s+s^{\prime}=2 \delta$, we get easily

$$
2 a_{\xi^{\prime}}=\left|\Lambda^{\prime}-\delta\right|^{2}-\left|\xi^{\prime}-\delta\right|^{2} .
$$

Thus we get

$$
2 \Delta^{-}=\left(\left|\Lambda^{\prime}-\delta\right|^{2}-\left|\xi^{\prime}-\delta\right|^{2}\right) 1
$$

on $U_{\xi^{\prime}}$.

We shall establish a condition on the weight $\xi^{\prime}$ in order that the equality $\left|\Lambda^{\prime}-\delta\right|=\left|\xi^{\prime}-\delta\right|$ holds (see Proposition 10.1 below). We denote as above by $\Sigma$ the set of (non-zero) roots of $\mathrm{g}^{c}$ and by $\Sigma_{+}$(resp. $\Sigma_{-}$) the set of positive (resp. negative) roots. For a subset $\Phi$ of $\Sigma$ we denote by $\langle\Phi\rangle$ the sum of the roots belonging to $\Phi$. Let $W$ be the Weyl group of $\mathrm{g}^{C}$ and for an element $T$ of $W$, we put

$$
\Phi_{T}=T\left(\Sigma_{-}\right) \cap \Sigma_{+} \cdot
$$

We have then the quality

$$
T \delta=\delta-\left\langle\Phi_{T}\right\rangle
$$

(see [8]). Put

$$
W^{1}=\left\{T \in W ; \Phi_{T} \subset \Psi\right\},
$$

$\Psi$ being the set of positive complementary roots. An element $T$ of $W$ belongs to $W^{1}$ if and only if $T^{-1}(\Theta) \subset \Theta$ (see [8]).

Let $W_{1}$ be the Weyl group of the semisimple Lie algebra $\left[\mathfrak{f}^{c}, \mathfrak{l}^{c}\right]$. In a natural way we may consider $W_{1}$ as a group of linear transforma- 
tions on the real vector space spanned by $\Sigma$ and we may identify $W_{1}$ with a subgroup of $W$. Then $W^{1}$ constitutes a complete set of representatives of right cosets of $W \bmod . W_{1}$, i.e. each element $T$ of $W$ is written uniquely in the form

$$
T=T_{1} T_{2} \quad\left(T_{1} \in W_{1}, T_{2} \in W^{1}\right)
$$

(see [8]). Under these notations, we have the following proposition.

Proposition 10. 1. (Kostant). Let

$$
F \otimes \wedge \mathfrak{n}^{+}=\Sigma U_{\xi^{\prime}}
$$

be a decompositiou of $F \otimes \wedge \mathfrak{n}^{+}$into direct sum of irreducible $\mathfrak{l}^{c}$-modules $U_{\xi^{\prime}}$, where $\xi^{\prime}$ denotes the lowest weight of the irreducible representation of ${ }^{C}$ in $U_{\xi^{\prime}}$. Let $\Lambda^{\prime}$ be the lowest weight of the irreducible representation $\rho$ of $\mathrm{g}^{c}$ in $F$. Then we have

$$
\left|\Lambda^{\prime}-\delta\right| \geqq\left|\xi^{\prime}-\delta\right|
$$

and the equality

$$
\left|\Lambda^{\prime}-\delta\right|=\left|\xi^{\prime}-\delta\right|
$$

holds if and only if there exists $T \in W^{1}$ such that

$$
\xi^{\prime}=T \Lambda^{\prime}+\left\langle\Phi_{T}\right\rangle .
$$

Moreover, the mapping $T \rightarrow \xi_{T}^{\prime}=T \Lambda^{\prime}+\left\langle\Phi_{T}\right\rangle$ is a bijection of $W^{1}$ onto the set of the lowest weights $\xi^{\prime}$ appearing in the decomposition (10.1) and satisfying (10.2).

Let $n(T)$ denote the number of the roots belonging to $\Phi_{T}$. Then a weight vector of $\xi_{T}^{\prime} i s, u p$ to a scalar multiple, of the form

$$
e_{T \Lambda^{\prime}} \otimes\left(\bigwedge_{\infty \in \Phi_{T}} X_{\infty}\right)
$$

where $e_{T \Lambda^{\prime}}$ is a weight vector of $T \Lambda^{\prime}$, and $U_{\xi^{\prime} T}$ is therefore a subspace of $F \otimes \bigwedge^{n(T)} \mathfrak{n}^{+}$. Consequently the space

$$
\sum_{T \in W^{1}, n(T)=q} U_{\xi^{\prime}}
$$

coincides with the space of all harmonic cocycles of $C^{q}\left(\mathfrak{n}^{-}, F\right)$.

The proof of Proposition 10.1 is parallel to a proof, given by Cartier [4], of Kostant's main result in [8], so we may omitt it.

If $\rho$ is a trivial representation of $\mathrm{g}^{c}$, then the coboundary operator $d^{-}$of $C\left(\mathfrak{n}^{-}, F\right)$ reduces to 0 and so is the laplacian $\Delta^{-}$. We get therefore the following 
Proposition 10.2. (Kostant). The $\mathfrak{l}^{c}$-module $\bigwedge^{q} \mathfrak{n}^{+}$decomposes into a direct sum of irreducible $\mathfrak{l}^{C}-$ modules

$$
\bigwedge^{q} \mathfrak{n}^{+}=\sum_{T \in W^{1}, n(T)=q} Q_{<\mathbf{q}_{T}>}
$$

where $\left\langle\Phi_{T}\right\rangle$ is the lowest weight of the representation of $\mathfrak{l}^{c}$ in $Q_{<\Phi_{T}>}$.

We know that there exists one and only one element $R \in W$ such that $R\left(\Sigma_{+}\right)=\Sigma_{-}$. Let

$$
R=R_{1} R^{1}, \quad R_{1} \in W_{1}, \quad R^{1} \in W^{1} .
$$

Then we see easily that

$$
R_{1}(\Theta)=-\Theta
$$

Therefore the highest weights in $U_{\xi^{\prime}{ }_{T}}$ and $Q_{\left\langle\Phi_{T}>\right.}$ are $R_{1}\left(\xi_{T}^{\prime}\right)$ and $\left\langle R_{1} \Phi_{T}\right\rangle$ respectively and we write:

$$
\begin{aligned}
& F \otimes \bigwedge^{q} \mathfrak{n}^{+}=\sum_{T \in W^{1}, n(T)=q} U^{R_{1}\left(\xi^{\prime} T^{\prime}\right)}, \\
& \bigwedge^{q} \mathfrak{n}^{+}=\sum_{T \in W^{1}, n(T)=q} Q^{<R_{1}\left(\Phi_{T}\right)>} .
\end{aligned}
$$

We consider now the cochain complex $C\left(\mathfrak{n}^{+}, F\right)$ of the abelian Lie algebra $\mathfrak{n}^{+}$and define the laplacian $\Delta^{+}$as in the case of the cochain complex $C\left(\mathfrak{n}^{-}, F\right)$. Identifying $C\left(\mathfrak{n}^{+}, F\right)$ with $F \otimes \wedge \mathfrak{n}^{-}$, we get the following formula :

$$
2 \Delta^{+}=\rho(C) \otimes 1+\sum_{a}\left(\rho \otimes \mathrm{ad}_{-}\right)\left(Y_{a}\right)^{2}+\sum_{k}\left(\rho \otimes \operatorname{ad}_{-}\right)\left(\left[X_{\bar{k}}, X_{k}\right]\right) .
$$

We obtain also the following two propositions due to Kostant.

Proposition 10. 3. Let

$$
F \otimes \wedge \mathfrak{n}^{-}=\sum V^{\xi}
$$

be a decomposition of $F \otimes \wedge \mathfrak{n}^{-}$into direct sum of irreducible $\mathfrak{l}^{C}$-modules $V^{\xi}$, where $\xi$ denotes the highest weight of the irreducible representation of ${ }^{C}$ in $V^{\xi}$. Let $\Lambda$ be the highest weight of the irreducible representation $\rho$ of $\mathrm{g}^{C}$ in $F$. Then the laplacian $\Delta^{+}$leaves invariant each of the subspace $V^{\xi}$ and we have

$$
2 \Delta^{+}=\left(|\Lambda+\delta|^{2}-|\xi+\delta|^{2}\right) 1
$$

in $V^{\xi}$. Moreover we have

and the equality

$$
|\Lambda+\delta| \geqq|\xi+\delta|
$$

$$
|\Lambda+\delta|=|\xi+\delta|
$$


holds if and only if there exists $T \in W^{1}$ such that

$$
\xi=T \Lambda-\left\langle\Phi_{T}\right\rangle \text {. }
$$

The mapping $T \rightarrow \xi_{T}=T \Lambda-\left\langle\Phi_{T}\right\rangle$ is a bijection of $W^{1}$ onto the set of highest weights $\xi$ appearing in the decomposition (10.3) and satisfying the equality (10.4).

A weight vector of $\xi_{T}$ is, up to a scalar multiple, of the form

$$
e_{T \Lambda} \otimes\left(\bigwedge_{\omega \in \Phi_{T}} X_{-\infty}\right)
$$

where $e_{T \Lambda}$ is a weight vector of $T \Lambda$, and $V^{\xi_{T}}$ is a subspace of $F \otimes \bigwedge^{n(T)} \mathfrak{n}^{-}$. The space

$$
\sum_{t \in W^{1}, n(T)=p} V^{\xi_{T}}
$$

coincides with the space of all harmonic cocycles of $C^{p}\left(\mathfrak{n}^{+}, F\right)$.

Proposition 10.4. The $\mathfrak{l}^{C_{-}-m o d u l e} \bigwedge^{p} \mathfrak{n}^{-}$decomposes into a direct sum of irreducible $\mathfrak{l}^{\mathrm{C}}$-modules

$$
\bigwedge^{p} \mathfrak{n}^{-}=\sum_{T \in W^{1}, n(T)={ }_{p}} P^{-<\Phi_{T}>}
$$

where $-\left\langle\Phi_{T}\right\rangle$ is the highest weight of the representation of $\mathfrak{l}^{C}$ in $P^{\left.-<\Phi_{T}\right\rangle}$.

11. The operators $L^{\prime}$ and $L^{\prime \prime}$ in the module $F \otimes \wedge \mathfrak{n}^{-} \otimes \wedge \mathfrak{n}^{+}$

We define two operators $L^{\prime}$ and $L^{\prime \prime}$ in the vector space

$$
\mathscr{L}=F \otimes \wedge \mathfrak{n}^{-} \otimes \wedge \mathfrak{n}^{+}
$$

by putting

$$
\begin{aligned}
& L^{\prime}=\Delta^{+} \otimes 1 \\
& L^{\prime \prime}=t^{-1} \circ\left(\Delta^{-} \otimes 1\right) \circ t,
\end{aligned}
$$

where $t$ is the canonical isomorphism of $F \otimes \wedge \mathfrak{n}^{-} \otimes \wedge \mathfrak{n}^{+}$onto $F \otimes \wedge \mathfrak{n}^{+}$ $\otimes \wedge \mathfrak{n}^{-}$defined by $t(f \otimes u \otimes v)=f \otimes v \otimes u$. Put

$$
\mathscr{L}^{p, q}=F \otimes \bigwedge^{p} \mathfrak{n}^{-} \otimes \bigwedge^{q} \mathfrak{n}^{+}
$$

and

$$
\mathscr{H}^{p, q}=\left\{c \in \mathscr{L}^{p, q} ; L^{\prime} c=L^{\prime \prime} c=0\right\}
$$

Let

$$
F=\sum_{\mu} F^{\mu}
$$

be a decomposition of $F$ into a direct sum of irreducible $\mathfrak{l}^{C}$-modules, where $\mu$ denotes the highest weight of the irreducible representation of ${ }^{C}$ in $F$. Put 
We have then

$$
W^{1}(r)=\left\{T \in W^{1} ; n(T)=r\right\}
$$

$$
\mathscr{L}^{p, q}=\sum_{\mu} \sum_{S \in W^{1}(p)} \sum_{T \in W^{1}(q)} \mathscr{L}(\mu, S, T),
$$

where we put

$$
\mathscr{L}(\mu, S, T)=F^{\mu} \otimes P^{<-\Phi_{S}>} \otimes Q^{<R_{1}\left(\Phi_{T}\right)>} .
$$

We see easily that $\mathscr{L}(\mu, S, T)$ is invariant by $L^{\prime}$ and $L^{\prime \prime}$ and hence we have

$$
\mathcal{H}^{p, q}=\sum_{\mu} \sum_{S \in W^{1}(q)} \sum_{T \in W^{1}(q)} \mathcal{H}^{p, q}(\mu, S, T)
$$

where we put

$$
\mathscr{H}^{p, q}(\mu, S, T)=\mathcal{H}^{p, q} \cap \mathscr{L}(\mu, S, T) .
$$

Lemma 11. 1. Let $c$ be an element of $F^{\mu} \otimes Q^{<R_{1}\left(\Phi_{T}\right)>}$ and suppose $\Delta^{-} c=0$ and $c \neq 0$. Then we have $\mu=R_{1} T \Lambda^{\prime}$.

Proof. By the assumption of the lemma, the intersection of $F^{\mu} \otimes$ $Q^{<R_{1}\left(\Phi_{T}\right)>}$ with $\sum_{T^{\prime} \in W^{1}(q)} U^{R_{1} \xi^{\prime} T^{\prime}}(q=n(T))$ is not empty, and hence there exists $T^{\prime} \in W^{1}(q)$ such that $U^{R_{1} \xi^{\prime}{ }^{\prime \prime}}$ is contained in $F^{\mu} \otimes Q^{\left.<R_{1}\left(\Phi_{T}\right)\right\rangle}$. Then $\xi_{T^{\prime}}^{\prime}$ is a weight in $F^{\mu} \otimes Q^{\left.<R_{1}\left(\Phi_{T}\right)\right\rangle}$ and hence there exists a weight $\nu$ in $F^{\mu}$ and a subset $\Phi$ of $\Psi$ such that

$$
\xi_{T^{\prime}}^{\prime}=\nu+\langle\Phi\rangle \text {. }
$$

We get then $\nu=T^{\prime} \Lambda^{\prime}$ and $\Phi=\Phi_{T^{\prime}}\left([8]\right.$ or [4]). Since $T^{\prime^{-1}}(\Theta) \subset \Theta$ and $\left\langle T^{\prime} \Lambda^{\prime}, \beta\right\rangle=\left\langle\Lambda^{\prime}, T^{\prime-1} \beta\right\rangle$, we have $\left\langle T^{\prime} \Lambda^{\prime}, \beta\right\rangle \leqq 0$ for all $\beta \in \Theta$, because $\Lambda^{\prime}$ is the lowest weight of the representation $\rho$ of $\mathrm{g}^{C}$. It follows from this that $\nu=T^{\prime} \Lambda^{\prime}$ is the lowest weight in $F^{\mu}$ and hence we get $R_{1} T^{\prime} \Lambda^{\prime}=\mu$. On the other hand, since $\left\langle\Phi_{T^{\prime}}\right\rangle$ is the lowest weight in $Q_{\left\langle\Phi_{T^{\prime}}\right\rangle}$, we have $\left\langle\left\langle\Phi_{T^{\prime}}\right\rangle, \beta\right\rangle \leqq 0$ for $\beta \in \Theta$ and, as $\left\langle\Phi_{T^{\prime}}\right\rangle$ is a weight in $\left.Q_{\left\langle\Phi_{T}\right.}\right\rangle$, we get $\left\langle\Phi_{T^{\prime}}\right\rangle=\left\langle\Phi_{T}\right\rangle$. Since the correspondence $T \rightarrow\left\langle\Phi_{T}\right\rangle\left(T \in W^{1}\right)$ is oneto-one, we get $T=T^{\prime}$ and we have finally $\mu=R_{1} T \Lambda^{\prime}$.

Analogously we obtain

Lemma 11.2. Let $c$ be an element of $F^{\mu} \otimes P^{\left.-<\Phi_{S}\right\rangle}$ and suppose $\Delta^{+} c=0$ and $c \neq 0$. Then we have $\mu=S \Lambda$.

Lemma 11. 3. The space $\mathcal{H}^{p, q}(\mu, S, T)$ is different from (0) if and only if we have $\mu=S \Lambda=R_{1} T \Lambda^{\prime}$.

In fact, if $\mathcal{H}^{p, q}(\mu, S, T) \neq(0)$, we get $\mu=S \Lambda=R_{1} T \Lambda^{\prime}$ by Lemmas 11. 1 and 11.2. Conversely, if we have $\mu=S \Lambda=R_{1} T \Lambda^{\prime}$, the element $c=e_{\mu} \otimes\left(\Lambda_{a \in \Phi_{S}} X_{-\infty}\right) \otimes\left(\Lambda_{\omega \in R_{1}\left(\Phi_{T}\right)} X_{\omega}\right)$ belongs to $\mathcal{H}^{p, q}(\mu, S, T)$.

Let $\alpha$ be a root belonging to $\Psi$. Then $\alpha$ is a weight in the $C_{-}$ module $\mathfrak{n}^{+}$and so is $R_{1} \alpha$. Thus we have $R_{1}(\Psi)=\Psi$ : 
Lemma 11. 4. Let $S$ and $T$ be two elements of $W^{1}$ such that $S \Lambda=$ $R_{1} T \Lambda^{\prime}$ holds. Let $\alpha \in \Psi$. If $\alpha \in \Phi_{S} \cap R_{1}\left(\Phi_{T}\right)$ or $\alpha \equiv \Phi_{S} \cup R_{1}\left(\Phi_{T}\right)$, then we have $\left\langle\Lambda, S^{-1} \alpha\right\rangle=0$.

Proof. By the definition of $W^{1}, \Phi_{T}$ is a subset of $\Psi$ and $\Phi_{T}=$ $T\left(\Sigma_{-}\right) \cap \Psi$. Hence $R_{1} \alpha$ belongs to $\Phi_{T}$ if and only if $T^{-1} R_{1} \alpha<0$. Since $R_{1}^{2}=1$ and $R\left(\Sigma_{-}\right)=\Sigma_{+}$, we see that $\alpha$ belongs to $R_{1}\left(\Phi_{T}\right)$ if and only if $R T^{-1} R_{1} \alpha>0$. Analogously, we see that $\alpha$ belongs to $\Phi_{S}$ if and only if $S^{-1} \alpha<0$. Suppose that $\alpha \in \Phi_{S} \cap R_{1}\left(\Phi_{T}\right)$. Then $\langle S \Lambda, \alpha\rangle=\left\langle\Lambda, S^{-1} \alpha\right\rangle \leqq 0$, because $S^{-1} \alpha$ is negative and $\Lambda$ is the highest weight of the representation $\rho$. On the other hand, as we have $S \Lambda=R_{1} T \Lambda^{\prime}, \Lambda^{\prime}=R \Lambda, R_{1}^{2}=1$ and $R^{2}=1$, we have $\langle S \Lambda, \alpha\rangle=\left\langle\Lambda, R T^{-1} R_{1} \alpha\right\rangle \geqq 0$, because $R_{1} T^{-1} R \alpha$ is positive. Therefore we get $\left\langle\Lambda, S^{-1} \alpha\right\rangle=0$. Analogously we get $\left\langle\Lambda, S^{-1} \alpha\right\rangle$ $=0$ if $\alpha \bar{\in} \Phi_{S} \cup R_{1}\left(\Phi_{T}\right)$.

Proposition 11. 1. Suppose that $\langle\Lambda, \alpha\rangle>0$ for all positive roots $\alpha$. Then we have

$$
\mathcal{H}^{p, \boldsymbol{q}}=(0)
$$

for $p+q \neq N$.

Proof. By the assumption we have $\langle\Lambda, \beta\rangle \neq 0$ for any root $\beta \neq 0$. Let $S$ and $T$ be two elements of $W^{1}$ such that $S \Lambda=R_{1} T \Lambda^{\prime}$ holds. It follows then from Lemma 11.4 that we must have $\Phi_{S} \cup R_{1}\left(\Phi_{T}\right)=\Phi$ and $\Phi_{S} \cap R_{1}\left(\Phi_{T}\right)=\phi$. In particular, we get $n(S)+n(T)=N$. We see then from Lemma 11.3 that $\mathcal{H}^{p, q} \neq(0)$ implies $p+q=N$.

Proposition 11.2. Let $p_{\rho}$ be the number of roots $\alpha$ in $\Psi$ such that $\left\langle\Lambda^{\prime}, \alpha\right\rangle<0$. Then we have

for $p<p_{\rho}$.

$$
\mathscr{H}^{p, 0}=(0)
$$

Proof. Let $S$ be an element of $W^{1}$ such that $S \Lambda=R_{1} \Lambda^{\prime}$. (Note that $\left.W^{1}(0)=1\right)$. Let $\alpha \in \Psi$ and let $\left\langle\Lambda^{\prime}, \alpha\right\rangle<0$. We claim that $R_{1} \alpha$ belongs to $\Phi_{S}$. Indeed, we have $\left\langle R_{1} \Lambda^{\prime}, R_{1} \alpha\right\rangle\left\langle 0\right.$ and $R_{1} \Lambda^{\prime}=S \Lambda$, whence $\left\langle\Lambda, S^{-1} R_{1} \alpha\right\rangle$ $<0$. Therefore $S^{-1} R_{1} \alpha$ is negative and this implies $R_{1} \alpha \in \Phi_{S}$. It follows then that the number $n(S)$ of roots belonging to $\Phi_{S}$ is not less than $p_{\rho}$. We see from Lemma 11.3 that $\mathscr{H}^{p, 0} \neq(0)$ implies $p \geqq p_{\rho}$ and the proposition is proved.

Analogously we get the following proposition which is identical with Theorem 8. 1.

Proposition 11. 3. Let $q_{\rho}$ be the number of roots $\alpha$ in $\Psi$ such that $\langle\Lambda, \alpha\rangle>0$. Then we have 
for $q<q_{\rho}$.

$$
\mathscr{H}^{0, \boldsymbol{q}}=(0)
$$

\section{Theorems on the cohomology groups $H^{p, q}(\Gamma, X, \rho)$}

Let now $\eta$ be an element of $C^{p, q}\left(\mathfrak{g}, \mathfrak{f} ; \mathcal{F}^{\prime}\right)$. For a point $x$ of $\Gamma \backslash G$ we define a mapping $\eta \rightarrow \eta(x)$ of $C^{p, q}\left(\mathfrak{g}^{c}, \mathfrak{l}^{C} ;\right.$ F $)$ into $\mathscr{L}^{p, q}=F \otimes \bigwedge^{p} \mathfrak{n}^{-} \otimes \bigwedge^{q} \mathfrak{n}^{+}$ by putting

$$
\left.\eta(x)=\sum_{i_{1}<\cdots<i_{p}} \sum_{j_{1}<\cdots j_{q}} \eta_{i_{1} \cdots i_{p} j_{1} \cdots \bar{j}_{q}}(x) \otimes\left(X_{\bar{i}_{1}} \wedge \cdots \wedge X_{\bar{i}_{p}}\right) \otimes X_{j_{1}} \wedge \cdots \wedge X_{j_{q}}\right) .
$$

Note that $\eta_{i_{1} \cdots i_{p} j_{1} \cdots j_{q}}=\eta\left(X_{i_{1}}, \cdots, X_{i_{p}}, X_{\bar{j}_{1}}, \cdots, X_{\bar{j}_{q}}\right)$ is an $F$-valued $C^{\infty}$-function on $\Gamma \backslash G$. It follows from (5.4) and (5.6) that we have

$$
\begin{aligned}
& \left(\Delta_{\rho}^{\prime} \eta\right)(x)=L^{\prime}(\eta(x)), \\
& \left(\Delta_{\rho}^{\prime \prime} \eta\right)(x)=L^{\prime \prime}(\eta(x)) .
\end{aligned}
$$

Therefore if $\eta$ is a harmonic cocycle in $C^{p, q}\left(\mathfrak{g}^{c}, \mathfrak{l}^{C} ; \mathcal{F}\right)$, then we have $\eta(x) \in \mathcal{H}^{p, q}$ for all $x \in \Gamma \backslash G$.

We get then from Propositions 11.1 and 11.2 the following vanishing theorems.

Theorem 12.1. Let $\rho$ be an irreducible representation of $G$ in a complex vector space $F$ and let $\Lambda$ be the highest weight of $\rho$. Suppose that $\langle\Lambda, \alpha\rangle>0$ for all positive roots $\alpha$ of $\mathrm{g}^{c}$. Then the cohomology group $H^{p, q}(\Gamma, X, \rho)$ vanishes for $p+q \neq N$.

This theorem generalizes a part of the main theorem of [10].

Theorem 12.2. The notions being as in Theorem 12.1, let $\Lambda^{\prime}$ be the lowest weight of $\rho$. Let $p_{\rho}$ be the number of roots $\alpha$ in $\Psi$ such that $\left\langle\Lambda^{\prime}, \alpha\right\rangle<0$. Then the cohomology group $H^{p, 0}(\Gamma, X, \rho)$ vanishes for $p<p_{\rho}$.

We can also derive Theorem 8.2 from Proposition 11.3.

Suppose now $\Gamma$ acts freely on $X$. Then we have

$$
\sum_{r=0}^{2 N}(-1)^{r} \operatorname{dim}_{C} H^{r}(\Gamma, X, \rho)=\operatorname{dim}_{\boldsymbol{C}} F \cdot E(\Gamma \backslash X) \quad(\rho: \text { arbitrary })
$$

where $E(\Gamma \backslash X)$ denotes the Euler characteristic of the complex manifold $\Gamma \backslash X$ (see [10]). By a theorem of Hirzebruch [6], we have

$$
E(\Gamma \backslash X)=\chi(\Gamma \backslash X) E\left(X_{u}\right)
$$

where $\chi(\Gamma \backslash X)$ is the arithmetic genus of $\Gamma \backslash X$ and $E\left(X_{u}\right)$ is the Euler characteristic of the compact form $X_{u}$ of $X$. Furthermore, if $X$ is irreducible, Hirzebruch [5] has given the following formula.

$$
E(\Gamma \backslash X)=(-\pi)^{-N} d_{N} v(\Gamma \backslash X),
$$


where $v(\Gamma \backslash X)$ denotes the total volume of $\Gamma \backslash X$ measured by the volume element associated to the Bergman metric on $X$ and

$$
d_{N}=\frac{\Pi_{\omega \in \Psi}\langle\delta, \alpha\rangle}{\left(2\left\langle s, \gamma_{1}\right\rangle\right)^{N}} \cdot E\left(X_{u}\right)
$$

Here $\delta=\frac{1}{2} \sum_{\alpha>0} \alpha, s=\sum_{\alpha \in \Psi} \alpha$ and $\gamma_{1}$ is the unique simple root belonging to $\Psi$. Since $2\left\langle s, \gamma_{1}\right\rangle=1$ by Lemma 4.2 , we get

$$
\left.d_{N}=\Pi_{\omega \in \Psi}\langle\delta, \alpha)\right\rangle \cdot E\left(X_{u}\right) .
$$

Therefore, we have

$$
\chi(\Gamma \backslash X)=(-\pi)^{-N} \Pi_{\omega \in \boldsymbol{\Psi}}\langle\delta, \alpha\rangle \cdot v(\Gamma \backslash X) .
$$

On the other hand, by Weyl's formula we have

$$
\operatorname{dim}_{c} F=\Pi_{\alpha>0} \frac{\langle\Lambda+\delta, \alpha\rangle}{\langle\delta, \alpha\rangle}
$$

Combining these formulae, we get from Theorem 12.1 the following theorem.

Theorem 12. 3. Under the same notations and assumptions as in Theorem 12.1, suppose that $X$ is irreducible and that $\Gamma$ acts freely on $X$. Then we have

$$
\begin{gathered}
H^{r}(\Gamma, X, \rho)=(0) \quad(r \neq N) \\
\operatorname{dim}_{C} H^{N}(\Gamma, X, \rho)=(-\pi)^{-N} \frac{\Pi_{\alpha>0}\langle\Lambda+\delta, \alpha\rangle}{\Pi_{\omega \in \Theta}\langle\delta, \alpha\rangle} E\left(X_{u}\right) v(\Gamma \backslash X) .
\end{gathered}
$$

OsAKA UNIVERSITY

\section{Bibliography}

[1] A. Borel: On the curvature tensor of the hermitian symmetric manifolds, Ann. of Math. 71 (1960), 508-521.

[2] A. Borel and F. Hirzebruch: Characteristic classes and homogeneous spaces, Part I, Amer. J. Math. 80 (1958), 459-538.

[3] E. Calabi and E. Vesentini : On compact, locally symmetric Kähler manifolds, Ann. of Math. 71 (1960), 472-507.

[4] P. Cartier: Remarks on "Lie algebra cohomology and the generalized BorelWeil theorem" by B. Kostant, Ann. of Math. 74 (1961), 388-390.

[5] F. Hirzebruch: Characteristic numbers of homogeneous domains, Seminars on Analytic Functions, Vol. 2, Princeton, 1957. 92-104. 
[6] F. Hirzebruch: Automorphe Formen und der Satz von Riemann-Roch, Symposium internacional de Topologia Algebraica, Mexico, 1958. 129-144.

[7] M. Ise: Generalized automorphic forms and certain holomorphic vector bundles, Amer. J. Math. 86 (1964), 70-108.

[8] B. Kostant: Lie algebra cohomology and the generalized Borel-Weil theorem, Ann. of Math. 74 (1961), 329-387.

[9] Y. Matsushima and S. Murakami : On vector bundle valued harmonic forms and automorphic forms on symmetric riemannian manifolds, Ann. of Math. 78 (1963), 365-416.

[10] Y. Matsushima and G. Shimura: On the cohomology groups attached to certain vector valued differential forms on the product of the upper half planes, Ann. of Math. 78 (1963), 417-449.

[11] M. S. Raghunathan: On the first cohomology of discrete subgroups of semisimple Lie groups, Amer. J. Math. 87 (1965), 103-139.

[12] M. Kuga: Lecture note, University of Chicago, 1963-64. 
